\title{
A Flexible Model of Term-Structure Dynamics of Commodity Prices: A Comparative Analysis with a Two-Factor Gaussian Model
}

\author{
Hiroaki Suenaga \\ School of Economics and Finance, Curtin University, GPO Box U1987, Perth, WA 6845, \\ AUSTRALIA. \\ Email: hiroaki.suenaga@cbs.curtin.edu.au
}

\begin{abstract}
This study compares two approaches to modeling a term structure of commodity prices. The first approach specifies the stochastic process of the underlying spot price and derives from the stipulated spot price dynamics valuation formulas of futures and other derivative contracts through no arbitrage. The second approach, as introduced by Smith (2005), is to model the dynamics of the entire futures curve directly by a set of common stochastic factors and to specify factor loadings by flexible functions of time-to-maturity and contract delivery month. Empirical applications of the models to four commodities (gold, crude oil, natural gas, and corn) reveal that the volatility of futures prices exhibits more complex dynamics than the pattern implied by the model stipulating a two-factor Gaussian process of the underlying spot price. Specifically, the flexible model of futures returns depicts the maturity effect and, particularly for the three consumption commodities, strong seasonal and cross-sectional variations in variance and covariances of concurrently traded contracts. Incorporating the depicted variance and covariance dynamics leads the flexible model of futures returns to suggest hedging strategies that are more effective than the strategies based on the conventional two-factor term-structure model.
\end{abstract}

Keywords: commodity prices, term-structure model, volatility, hedging 


\section{Introduction}

Recent increase in the level and volatility of oil, metals, and other primary commodity prices has created tremendous uncertainties for producers, consumers, and other traders of these commodities. Volatility of commodity prices also affects the national economy, both directly by altering revenue and expenditure on these commodities and indirectly by deferring new investments. In such circumstances, a better understanding of the stochastic properties of these commodity prices and tools to hedge against price risks becomes increasingly important for the smooth functioning of the commodity supply chain.

Stochastic dynamics of commodity prices and valuation of derivative contracts have long been studied in the field of financial economics. The standard approach in this literature is to specify the stochastic process of the underlying asset, usually the spot price of the commodity under investigation, and derive from the stipulated process valuation formulas of futures and other derivative contracts whose payoff depends on the value of the underlying asset realized at the contract maturity date (see Hull, 2002). This approach dates back to Black and Scholes (1973) who derived pricing formulas of European options under the assumption that the underlying asset value (stock price) follows a geometric Brownian motion (GBM). While following the same approach, many studies modeling commodity price dynamics commonly specify one of the underlying stochastic factors to follow a mean-reverting (MR) process because, for many consumption commodities, demand and/or supply response forces unusually high or low prices to revert to the long-run equilibrium level (Schwartz, 1997).

Recent advances in this modeling approach have been attained through increasing the number of common stochastic factors and/or stipulating an increasingly complex stochastic process of each latent factor. ${ }^{1}$ These flexible models generally exhibit a better fit to the observed price data while maintaining the model parsimonious with pricing formulas of derivative contracts typically determined by a small number of parameters characterizing the stochastic dynamics of the underlying factors. However, it is often understated that the benefit of a parsimonious specification is gained at the cost of potentially large errors in approximating true stochastic dynamics of commodity prices. It has been widely acknowledged that, unlike stocks and other conventional financial assets, commodity prices exhibit complex dynamics. The theory of storage (e.g., Williams and Wright, 1991; Routledge et al., 2000) illustrate that, for a commodity with a significant storage cost, inter-temporal

${ }^{1}$ Lautier (2005) provides a comprehensive review on applications of term-structure models to various commodities. 
arbitrage establishes an equilibrium constellation of spot and futures prices along which the marginal benefit of current consumption is equal to or above the expected marginal benefit of storing a commodity for future consumption. The weak inequality stems from a nonnegativity of physical storage. If supply is ample relative to demand, inter-temporal arbitrage induces positive inventory up to a point where the two prices differ by the cost of carry. In this case, the convenience yield, representing the implicit revenue from holding a physical asset, is close to zero. In contrast, when supply is scarce, discretionary inventory is driven to zero and the marginal benefit of current consumption exceeds the marginal benefit of future consumption due to high convenience yield. In this case, speculative storage plays a minor role in price determination and the inter-temporal price linkage breaks. This discontinuity in the inter-temporal price link means that price correlations across concurrently traded contracts vary by season. Many commodities also exhibit pronounced seasonality in price and volatility, reflecting seasonality in the underlying demand and/or supply. Volatility tends to be high in the period of tight demand-supply balance because market shocks of even a small magnitude can cause a large price swing. It is also expected that volatility is inversely related to inventory because demand and supply shocks can be absorbed through adjusting inventory. Stochastic processes of the spot price and other underlying factors stipulated in many models of commodity price dynamics, even recently developed complex models, are often too simple to induce a futures price formula that replicates the complex dynamics of commodity futures prices implied by the theory of storage.

An alternative approach to modeling a term structure of commodity prices, as recently introduced by Smith (2005) and later extended by Suenaga and Smith (2011), is to model directly the dynamics of futures curve. In this model, daily futures returns is decomposed into a set of common stochastic factors affecting all futures returns and an idiosyncratic term. By modeling futures returns rather than a price level, the model does not specify seasonal or other deterministic variation in the underlying spot price. This model also avoids specifying stochastic dynamics of the underlying factors and imposes no a priori restriction on the factor loadings that connect underlying factors to observed futures returns. Rather, the model specifies factor loadings and the variance of the idiosyncratic term directly by flexible functions so that they can replicate highly non-linear price dynamics of commodities with significant storage costs and seasonality in demand or supply.

In this study, I compare the two approaches to the modeling of term structure of commodity prices; one specifying directly the dynamics of daily futures returns as a flexible function of common stochastic factors, and the other specifying the stochastic process of the 
underlying spot price. I apply the two models to futures price data from four commodity markets (crude oil, natural gas, gold, and corn). Results from this empirical analysis illustrate that the volatility of daily futures prices exhibits highly non-linear dynamics that cannot be induced by the stochastic process of the underlying spot price stipulated in the conventional two-factor term-structure model. Specifically, the flexible model of futures returns depicts a maturity effect and, particularly for the three consumption commodities, strong seasonality in both its levels and compositions among the two common stochastic factors and the idiosyncratic error. These features together create substantial seasonal and cross-sectional variation in the price correlations of concurrently traded contracts. Incorporating the depicted dynamics of price volatility and cross-contract correlations allows the flexible model of futures returns to suggest hedging strategies that are more effective than a strategy based on the conventional term-structure model specifying a two-factor Gaussian process for the underlying spot price.

The next section presents the two approaches to modeling a term structure of commodity prices. The section also presents a composite model in which the factor loadings are specified as in the conventional spot price-based approach, yet allows for a flexible variance structure of the idiosyncratic errors. Section 3 reports results from estimating the three models empirically with unbalanced panel data from four commodity markets. The results are compared across the models with an emphasis on seasonal and cross-sectional variations in the depicted price variance and cross-contract correlations. Section 4 considers the implication of the models for an optimal strategy to hedge price risk. Section 5 provides a synopsis of the findings to conclude the paper.

\section{Comparison of Two Modeling Approaches for Term-Structure of Commodity Futures}

This section presents a flexible model of futures curve dynamics in which daily futures returns is decomposed into a set of common latent factors and an idiosyncratic term. This model is then compared with the conventional term-structure model specifying stochastic dynamics of the underlying spot price and deriving pricing formulas of futures and other derivative contracts.

\subsection{A flexible model of commodity futures returns}

One approach to modeling commodity price dynamics, as introduced by Smith (2005) and later extended by Suenaga and Smith (2011), is to model directly the daily price changes 
of all concurrently traded futures contracts. ${ }^{2}$ In this approach, a daily return of futures contract is decomposed into the common latent factors and an idiosyncratic term. The model incorporates time varying conditional heteroskedasticity of latent factors and time and crosssectional variation in the factor loadings and idiosyncratic variances.

The model with two common factors is expressed in the following form,

$$
\Delta \ln F_{m, t}=\theta_{1}(m, d)\left(\mu_{1}+\varepsilon_{1, t}\right)+\theta_{2}(m, d)\left(\mu_{2}+\varepsilon_{2, t}\right)+\theta_{3}(m, d)\left(\mu_{3}+u_{m, t}\right)
$$

where $\Delta \ln F_{m, t}=\ln F_{m, t}-\ln F_{m, t-1}$ is the change from $t$ to $t+1$ of the log price of the futures contract that matures at $m . \varepsilon_{1, t}$ and $\varepsilon_{2, t}$ are the latent factors that affect all contracts traded on $t$, with $\mathrm{E}\left[\varepsilon_{t}\right]=0$ and $\mathrm{E}\left[\varepsilon_{t} \varepsilon_{t}^{\prime}\right]=I_{2} \forall t$ and $\mathrm{E}\left[\varepsilon_{t} \varepsilon_{s}^{\prime}\right]=0$ for $t \neq s$ where $\varepsilon_{t}=\left(\varepsilon_{1, t} \varepsilon_{2, t}\right)^{\prime} . u_{m, t}$ is the idiosyncratic error. It is assumed that $\mathrm{E}\left[u_{m, t}\right]=0$ and $\mathrm{V}\left[u_{m, t}\right]=1 \forall m$ and $t, \mathrm{E}\left[u_{m, s} u_{m, t}\right]=0$ $\forall s \neq t$, and, $\mathrm{E}\left[u_{n, t} u_{m, t}\right]=0 \quad \forall m \neq n$. In other words, $u_{m, t}$ represents shocks that are specific to the contract maturing at $m$ and uncorrelated serially and across concurrently traded contracts. $\theta_{1}(m, d)$ and $\theta_{2}(m, d)$ are the factor loadings determining the extent to which common shocks, $\varepsilon_{1, t}$ and $\varepsilon_{2, t}$, are reflected in the price change of the contract maturing at $m$, and $\theta_{3}(m, d)$ determines the standard deviation of the shock specific to the contract maturing at $m$. In model (1), three coefficients $\mu_{i}$ are included to allow for a potentially non-zero deterministic change in the log futures price. They are multiplied by the associated $\theta_{i}(m, d)$ function so that they are interpreted as representing the forward premium associated with the two common factors $(i=1,2)$ and idiosyncratic error $(i=3) .^{3}$

The three terms, $\theta_{i}(m, d)$ for $i=1,2$, and 3 , are specified as deterministic functions of contract delivery month $(m)$ and time to delivery of the contract $(d=m-t)$,

$$
\theta_{i}(m, d)=\exp \left[a_{i, m, 0}+a_{i, m, 1} d+\sum_{k=1}^{K}\left(a_{i, m, 2 k} \sin \left(\frac{2 \pi k d}{d_{\max }}\right)+a_{i, m, 2 k+1} \cos \left(\frac{2 \pi k d}{d_{\max }}\right)\right)\right]
$$

\footnotetext{
${ }^{2}$ The model as originally introduced by Smith (2005) is named as the Partially Overlapping Time-Series or "POTS" model for it is developed for the analysis of commodity futures return data, which usually forms a partially overlapping time series or an unbalanced panel. In this paper, I refer to the model defined in (1)-(3) as a "flexible model of futures returns" or simply "flexible model" because the other two models examined in this paper are also applied to partially overlapping time-series data.

${ }^{3}$ The estimates of the three coefficients $\mu_{i}$ are very small for all four commodities examined in this paper. Restricting these coefficients equal to zero does not alter the results presented in Sections 3 and 4 .
} 
where $d_{\max }$ is the maximum days to maturity for which the model is estimated. Specification (2) allows the three terms $\theta_{i}(m, d)$ to be a flexible function of time-to-maturity $(d)$ and permits this function to vary by contract delivery month $(m)$. The combination of $m$ and $d$ uniquely identifies a trade date $(t)$ in the year through $d=m-t$. Thus, specification (2) also captures seasonal variation in the factor loadings and the idiosyncratic variance. The function, $\theta_{i}(m, d)$, becomes more flexible with the number of trigonometric terms $(K)$. Although this extra flexibility allows a better fit to the observed data, it also makes the model more sensitive to extreme observations. In the empirical estimation of the model in Section 3, I set $K=3$ so that the model is flexible enough to capture seasonality and maturity effects while avoiding excess sensibility to extreme observations.

The unconditional variance of daily $\log$ futures returns is given by $\mathrm{V}\left[\Delta \ln F_{m, t}\right]=\sum_{i=1}^{3} \theta_{i}(m, d)^{2}$. Thus, the model can replicate potentially very complex dynamics in the variance of $\log$ futures returns and its composition among the three components. Furthermore, the two latent factors affect all contract prices whereas the idiosyncratic errors are uncorrelated across contracts. Therefore, correlations across concurrently traded contracts are determined by the share of the variance attributable to the two common factors. Specification (2) allows these cross-contract correlations to vary by season and across contracts.

For identification, the constraint is imposed as $a_{2, m, 0}=-\sum_{k=1}^{K} a_{2, m, 2 k+1}-a_{2, m, 1} d_{\max }-10$ so that $\theta_{2}\left(m, d_{\max }\right) \simeq 0$ for all $m$. That is, the loading of the second factor is close to zero at the maximum days to maturity. The condition is equivalent to the one used in Schwartz and Smith (2000), which allows the two factors to be interpreted as representing the long-term (LT) and short-term (ST) factor, respectively. ${ }^{4}$

The conditional variance of latent factors $\varepsilon_{t}$ is specified by a bivariate $\operatorname{GARCH}(1,1)$ model in a diagonal BEKK specification (Engle and Kroner, 1985),

$$
\begin{aligned}
& \mathrm{E}\left[\varepsilon_{t} \varepsilon_{t}^{\prime} \mid \mathfrak{J}^{t-1}\right]=\mathbf{H}_{t} \\
& \mathbf{H}_{t}=\boldsymbol{\Omega}+\boldsymbol{\beta} \mathbf{H}_{t-1} \boldsymbol{\beta}^{\prime}+\boldsymbol{\alpha E}\left[\varepsilon_{t-1} \varepsilon_{t-1}{ }^{\prime} \mid \mathfrak{J}^{t-1}\right] \boldsymbol{\alpha}^{\prime}
\end{aligned}
$$

\footnotetext{
${ }^{4}$ In the two-factor model of Schwartz and Smith (2000), the loading of the short-term factor in the futures price equation is given by $\exp (-\kappa \tau)$ where $\kappa$ and $\tau$ represents the mean-reversion coefficient and time-to-maturity, respectively. The value of this loading decreases exponentially and converges to zero with $\tau$, given $\kappa>0$. The two-factor model of Sorensen (2002) reviewed in Section 2.2 shares this property.
} 
where $E\left[\cdot \mid \mathfrak{I}^{t-1}\right]$ denotes that the expectation is conditional on the set of the information available at $t-1, \alpha$ and $\beta$ are 2 by 2 diagonal matrices of parameters, and $\Omega$ is a 2 by 2 diagonal matrix whose values are determined by $\boldsymbol{\Omega}=I_{2}-\boldsymbol{\beta} \boldsymbol{\beta}^{\prime}-\boldsymbol{\alpha} \boldsymbol{\alpha}^{\prime}$ due to $E\left[\varepsilon_{t} \varepsilon_{t}^{\prime}\right]=I_{2}$. This condition is required for the identification of the factor loadings $\theta_{i}(m, d)$ for $i=1$ and 2 .

Since the values of the two common factors are not observable, the coefficients in the model defined in (1)-(3) are estimated through the Kalman filter (Hamilton, 1994). The measurement equation is formulated by stacking all prices observed on day $t$,

$$
\Delta \mathbf{l n} \mathbf{F}_{t}=\mathbf{C}_{t}+\Theta_{1, t} \varepsilon_{1, t}+\Theta_{2, t} \varepsilon_{2, t}+\Theta_{3, t} \mathbf{u}_{t}
$$

where $\Delta \ln F_{t}$ is an $n_{t}$ by one column vector of the daily log futures returns observed on day $t$ with $n_{t}$ representing the number of futures contracts traded on $t, \mathbf{C}_{t}$ is an $n_{t}$ by one column vector of constants with its $j$-th element given by $\sum_{i=1}^{3} \theta_{i}\left(m_{j}, d_{j}\right) \mu_{i}, \Theta_{i, t}$ is an $n_{t}$ dimensional diagonal matrix with $\theta_{i}\left(m_{j}, d_{j}\right)$ on its $j$-th diagonal element, and $\mathbf{u}_{t}$ is an $n_{t}$ by one column vector with $u_{j, t}$ on its $j$-th element.

The two state variables are serially uncorrelated whereas the conditional variance of latent factors $\varepsilon_{t}$ is serially related through the GARCH process in (3). The conditional expectation in the second equation of (3) is,

$$
\mathrm{E}\left[\varepsilon_{t-1} \varepsilon_{t-1}{ }^{\prime} \mid \mathfrak{J}^{t-1}\right]=\varepsilon_{t-1 \mid t-1} \varepsilon_{t-1 \mid t-1}{ }^{\prime}+P_{t-1 \mid t-1}
$$

where $\varepsilon_{t-1 \mid t-1}=\mathrm{E}\left[\varepsilon_{t-1} \mid \Im^{t-1}\right]$ and $P_{t-1 \mid t-1}=\mathrm{E}\left[\left(\varepsilon_{t-1}-\varepsilon_{t-1 \mid t-1}\right)\left(\varepsilon_{t-1}-\varepsilon_{t-1 \mid t-1}\right)^{\prime} \mid \mathfrak{J}^{t-1}\right]$, which are obtained through the Kalman filter.

The model defined in (1)-(3) follows an approach similar to the one introduced by Heath et al. (1992) for modeling the term structure of interest rates; they both specify the dynamics of futures prices (forward rates) directly by a set of common latent factors. Theoretically, these factor models fit perfectly to the observed futures price changes if they include as many latent factors as the number of futures prices observed per day and impose no restrictions on the values of factor loadings. In practice, empirical models include only a small number of factors and impose certain parametric structures on the factor loadings. For example, Cortazar and Schwartz (1994), in their analysis of commodity contingent claims, include only 
three factors and plot the factor loadings as a function of time-to-maturity only. These restrictions create a discrepancy between the model's implied and observed price movement. Previous studies often do not model explicitly this residual component. The model defined in (1)-(3) differs from Cortazar and Schwartz (1994) in these regards; it specifies the two factor loadings as flexible parametric functions of time-to-maturity and contract delivery month. It also models parametrically the idiosyncratic error with its variance also specified by a flexible function. These specifications together allow the model to depict complex seasonal and crosssectional variations in the variance and covariances of concurrently traded contract prices.

\subsection{A conventional two-factor term-structure model of commodity prices}

A conventional approach to modeling the term structure of commodity prices is to specify the stochastic processes of the spot price of the commodity and to derive from the stipulated spot price dynamics pricing formulas of futures and other derivative contracts. For example, the following two-factor Gaussian model is commonly considered for the analysis of various commodities with seasonality in demand and/or supply, ${ }^{5}$

$$
\begin{aligned}
& \ln S_{t}=f(t)+x_{t}+z_{t} \\
& d x_{t}=\mu d t+\sigma_{x} d w_{x} \\
& d z_{t}=-\kappa z_{t} d t+\sigma_{z} d w_{z} \\
& d w_{x} d w_{z}=\rho d t
\end{aligned}
$$

where $S_{t}$ is the spot price at period $t, f(t)$ is the seasonal mean price that is a deterministic function of time, $x_{t}$ and $z_{t}$ are the state variables representing, respectively, the long-term (LT) and short-term (ST) deviation from the seasonal mean price, $d w_{x}$ and $d w_{z}$ are the increment to the standard Brownian motion that are correlated through $d w_{x} d w_{z}=\rho d t$, and $\mu, \kappa, \sigma_{x}$, and $\sigma_{z}$ are parameters determining, respectively, drift rate, mean reversion rate, and diffusion rate of the two stochastic factors.

The price in period $t$ of the futures contract that matures at $T$ is obtained as the period $t$ conditional expectation, under the risk neutral probability measure, of the spot price at $T$. It

\footnotetext{
${ }^{5}$ Model (4) has been considered, for example, for the analysis of electricity (Lucia and Schwartz, 2002), natural gas (Manoliu and Tompaidis, 2002), and agricultural commodity futures, such as corn, wheat, and soybean (Sorensen, 2002). Gibson and Schwartz (1990) and Nielsen and Schwartz (2004) also consider a two-factor model in analyzing oil and copper, yet they parameterize the dynamics of two factors differently from (4) so that the two factors are interpreted as representing spot price and convenience yield factor. Schwartz and Smith (2000) show that these models are identical to model (4) aside from the absence of seasonal variation in mean price.
} 
can be shown that, for the spot price following the process (4), the price of this futures contract is obtained as, ${ }^{6}$

$$
\ln F(t, T)=f(T)+A(\tau)+x_{t}+z_{t} e^{-\kappa \tau}
$$

where $A(\tau)=\left(\mu-\lambda_{x}+\frac{\sigma_{x}^{2}}{2}\right) \tau+\frac{\sigma_{z}^{2}\left(1-e^{-2 \kappa \tau}\right)}{4 \kappa}+\frac{\left(\rho \sigma_{x} \sigma_{z}-\lambda_{z}\right)\left(1-e^{-\kappa \tau}\right)}{\kappa}, \tau=T-t$ is the time-tomaturity, and two coefficients $\lambda_{x}$ and $\lambda_{z}$ represent the market prices of risk associated with the corresponding stochastic factor.

The set of parameters defining model (4), $\Omega=\left\{\kappa, \sigma_{x}, \sigma_{z}, \lambda_{x}, \lambda_{z}, \rho\right\}$, is usually estimated with futures price data. To fit equation (5) into multiple prices with different maturity dates observed per day, an error term is added to the right-hand side of (5), which makes the values of the two factors $x$ and $z$ unidentifiable. Thus, the model is commonly estimated with a filtering method. In state-space form, the model is presented as,

$$
\begin{aligned}
& \ln F\left(t, T_{i}\right)=f\left(T_{i}\right)+A\left(\tau_{i}\right)+x_{t}+z_{t} e^{-\kappa \tau_{i}}+u_{T_{i}, t} \\
& z_{t}=e^{-\kappa} z_{t-1}+v_{1, t} \\
& x_{t}=\mu+x_{t-1}+v_{2, t}
\end{aligned}
$$

where $T_{i}$ is the maturity date of $i$-th contract $\left(i=1, \ldots, n_{t}\right)$ observed on day $t, \mathbf{v}_{t}=\left(v_{1, t} v_{2, t}\right)^{\prime}$ is serially uncorrelated and identically distributed with $\mathbf{v}_{t} \sim \mathrm{N}(0, \mathbf{H})$ and $\mathbf{H}$ is the symmetric matrix with $\sigma_{x}^{2}$ and $\sigma_{z}^{2}$ on the main diagonal and $\rho \sigma_{x} \sigma_{z}$ off diagonal. In (6), $u_{T, t}$ is the measurement error representing errors in reporting prices or factors affecting futures prices that are not accounted for by the two common factors. It is commonly assumed that $\mathrm{E}\left[u_{T, t}\right]=0 \quad \forall t, T, \quad \mathrm{E}\left[u_{T_{1}, s} u_{T_{2}, t}\right]=0$ for $s \neq t$ and/or $T_{1} \neq T_{2}$. Thus, econometrically, $u_{T, t}$ represents the idiosyncratic error that is uncorrelated serially and contemporaneously across contracts. It is also commonly assumed that $\mathrm{V}\left[u_{T, t}\right]=\sigma_{T}^{2} \forall t$. That is, the variance is allowed to vary by the contract maturity date $(T)$ but not by trade date $(t)$ or time-to-maturity $(T-t)$.

${ }^{6}$ Futures price formula (5) assumes constant market prices of risk. See, for example, Sorensen (2002) for details. 


\subsection{Model comparison}

A major difference between conventional term-structure models of commodity prices and the flexible model of futures returns defined in (1)-(3) is that the former specifies the dynamics of price level whereas the latter specifies the dynamics of price return. By modeling price returns rather than level, the flexible model does not specify seasonality and other deterministic variations in the underlying spot price that result from demand/supply seasonality and other characteristics of the underlying commodity. ${ }^{7}$ Thus, the model is free from bias in specifying such deterministic price variation.

To compare the two models in further detail, take the first difference of the futures price formula in (7),

$$
\Delta \ln F_{T, t}=\ln F_{T, t}-\ln F_{T, t-1}=B(\tau)+v_{2, t}+e^{-\kappa \tau} v_{1, t}+\Delta u_{T, t}
$$

where $\quad B(\tau) \equiv \lambda_{x}+\left(\frac{\lambda_{z}-\rho \sigma_{x} \sigma_{z}}{\kappa}\right) e^{-\kappa \tau}\left(1-e^{-\kappa}\right)-\frac{\sigma_{x}^{2}}{2}-\frac{\sigma_{z}^{2} e^{-2 \kappa \tau}}{4 \kappa}\left(1-e^{-2 \kappa}\right) \quad$ and $\quad \Delta u_{T, t}{ }^{i i d} \sim \mathrm{N}\left(0,2 \sigma_{T}^{2}\right)$ because $\Delta u_{T, t}=u_{T, t}-u_{T, t-1}$ and $u_{T, t} \stackrel{i i d}{i} \mathrm{~N}\left(0, \sigma_{T}^{2}\right) \forall t$.

Comparison of (8) and (1) reveals three major benefits of the flexible model over the conventional approach in modeling a term structure of commodity prices with the specified spot price dynamics. First, model (1) specifies the factor loadings by flexible functions for both the LT and ST factors. In contrast, the factor loadings are determined by a small number of parameters defining the stochastic dynamics of the underlying state variables in conventional term-structure models. Specifically, for the two-factor model (8), the loadings of the ST factor decrease exponentially with time-to-maturity at an identical rate for all contracts, whereas those of the LT factor are constant at unity for all contracts throughout the trading horizon. Second, the flexible model (1) specifies the variance of the idiosyncratic error by a flexible function of time-to-maturity and allows this function to vary across contract delivery months. In contrast, conventional term-structure models impose a simplistic structure on the variance of the measurement error $u_{T, t}$ with the variance allowed to vary only by the delivery month of contract but not by time-to-maturity. Third, the innovations to

\footnotetext{
${ }^{7}$ These deterministic price variations correspond to the seasonal mean price and deterministic trend (denoted as $f(T)$ and $\mu$ ) in the conventional term-structure model (6). First differencing eliminates these terms and leaves only the innovation errors $\left(v_{1}\right.$ and $\left.v_{2}\right)$ on the right-hand side of (8). The stochastic dynamics of the two state variables still remain in (8), yet only implicitly by restricting functional forms of the factor loadings.
} 
the state variables, $v_{i, t}(i=1,2)$, are specified to follow a bivariate GARCH process in the flexible model (1) while they are assumed homoskedastic in the conventional term-structure model (8).

Strong restrictions imposed on the stochastic dynamics of the underlying factors and the variance of the measurement error potentially lead conventional term-structure models to draw an erroneous portrait of price volatility of a storable commodity with demand and/or supply seasonality. In particular, the model in (6) and (8) stipulates that the variance attributable to the two common factors increases exponentially as the contract approaches maturity whereas the variance of the measurement error does not vary with time-tomaturity. ${ }^{8}$ Consequently, the model implies that correlation across concurrently traded contracts decreases monotonically with time-to-maturity. By contrast, the flexible model defined in (1)-(3) allows for the magnitude of futures price change resulting from the common market shocks and that resulting from contract specific shocks to differ both by time-to-maturity and by contract delivery date. This flexibility allows the model to replicate highly non-linear dynamics of commodity prices expected by the theory of storage. The model also gives the same flexibility to the variance structure of the two common factors and that of the idiosyncratic error and thus avoids the magnitude and dynamics of the crosscontract correlation to be determined by the model specification.

In the next section, I estimate the flexible model of futures returns with empirical data from the four markets and compare its estimation results with the estimate of the two alternative models. The first model is the conventional two-factor model defined in (5)-(7). I estimate the subset of the model parameters that appear on the model's first difference form (8), which is directly comparable to the flexible model. Since first differencing eliminates the deterministic variation in mean price level and deterministic variations in the two common factors, the comparison signifies the adequacy of the parsimonious specifications imposed in the conventional two-factor model on the stochastic dynamics of the underlying factors (as determinants of the factor loadings) and the variance of the measurement error. The second alternative model is the composite model in which factor loadings are specified as in the conventional two-factor model (hence imposing a restrictive specification on the stochastic dynamics of the latent factors), whereas the variance of the measurement error is specified by a flexible function as in (2). That is,

\footnotetext{
${ }^{8}$ In other words, of the three components comprising the daily futures returns in model (8), the ST factor is only the component that can vary by time-to-maturity, and the measurement error is only the component that allows variation across contracts with different maturity dates.
} 


$$
\Delta \ln F_{T, t}=B(\tau)+v_{2, t}+e^{-\kappa \tau} v_{1, t}+\theta_{3}(m, d) u_{T, t}
$$

where $\theta_{3}(m, d)$ is as defined in (2). This model nests the two-factor model in (4)-(7). It is expected that the flexible specification of the variance of measurement error captures complex volatility dynamics, albeit partially.

\section{Data and Estimation}

This section empirically estimates the three models reviewed in Section 2 with the unbalanced panel data from four commodity markets. The section starts with the description of the data examined and then reports results from estimating the three models with emphasis on their implied volatility dynamics.

\subsection{Data}

The three models presented in Section 2 are estimated with the data from the markets for the following four commodities with varying characteristics:

- Natural gas - consumption good with strong seasonality in demand,

- Corn - consumption good with strong seasonality in supply,

- Crude oil - consumption good with very weak seasonality in demand and supply, and

- Gold - investment good with virtually no seasonality either in demand or supply.

The models are estimated using data on daily settlement prices of futures contracts traded at the New York Mercantile Exchange (crude oil, natural gas, and gold) and Chicago Board of Trade (corn). The data analysed in this paper are from the period between 1984/1/1 and 2007/12/31 for corn and gold, 1984/4/1 and 2007/12/31 for crude oil, and 1991/4/1 and 2007/12/31 for natural gas. For each contract, daily prices to the last trading day of the contract are used for analysis. ${ }^{9}$ Since long-dated contracts do not trade actively, contracts of more than twelve months to maturity are excluded from the analysis, except that contracts up to eighteen months to maturity are analyzed for corn..$^{10}$ Excluding these observations leaves

\footnotetext{
${ }^{9}$ Crude oil and natural gas contracts cease trading before the delivery month whereas corn and gold, contracts trade into the delivery month. See exchange's website (www.cmegroup.com) for details in contract specifications.

${ }^{10}$ Corn exhibits strong supply seasonality with harvest usually arriving around September to early November. The theory of storage suggests that inter-temporal price link breaks at the end of crop year, creating potentially very complex price dynamics for the September (and December) contract at around
} 
70,800 prices among 307 contracts for crude oil, 52,780 prices among 223 contracts for natural gas, 43,820 prices among 168 contracts for gold, and 48,762 prices among 142 contracts for corn.

All three models are estimated by the method of maximum likelihood with the likelihood obtained through the Kalman filter as described in Section 2. I first estimate the conventional two-factor model (8) which is the most parsimonious of the three models. The composite model (9) differs from model (8) only in the specification of the variance of the measurement error. The model is estimated with the starting values of the coefficient vector $\mathbf{a}_{3}$ in $\theta_{3}\left(m, d ; \mathbf{a}_{3}\right)$ obtained by minimizing the sum of the squared differences between $\theta_{3}\left(m, d ; \mathbf{a}_{3}\right)^{2}$ and the squared residuals from the estimated model (8). Finally, for the flexible model (1), I obtaine the starting values in two steps: (i) calculate the variance attributable to each of the three components (LT and ST factor, and the idiosyncratic error) from the estimated composite model and (ii) find the values of each coefficient vector $\mathbf{a}_{i}(i=1, \ldots, 3)$ that minimize the sum of the squared differences between $\theta_{i}\left(m, d ; \mathbf{a}_{i}\right)$ and the predicted values of the corresponding component in the composite model calculated in step (i). Robustness is checked by estimating the model with different sets of starting values obtained by distributing fraction of the variance of the idiosyncratic error from the estimated composite model to the other two components in step (i) of the above two-step procedure.

\subsection{Estimation results: Model specification}

Table 1 summarizes the results from the specification test. It shows that, for all four commodities considered, the flexible model of futures returns is preferred to the other two models, and the composite model is preferred to the conventional two-factor model according to both the Akaike and Schwarz Information Criteria. The results provide strong evidence that the conventional two-factor model stipulating restrictive structures on the factor loadings and the variance of the measurement errors is not supported empirically for all four commodities. Surprisingly, the model is not supported even for gold for which the storage cost is not significant and virtually no demand or supply seasonality exists.

12 months before maturity. I analyze the contracts as far as eighteen months to maturity to capture potentially very interesting price movements of these two contracts in this period. 


\subsection{Estimation results: Flexible model}

Figures 1 through 4 illustrate the results of estimating the flexible model of futures returns for the four commodities. These figures plot, for each contract delivery month: (a) the estimated loadings of the LT factor, (b) the loadings of the ST factor, (c) the standard deviation of the idiosyncratic shocks, and (d) the share of the total variance accounted for by the two common factors, which are all aligned by trade date. These components are calculated as $\theta_{i}\left(m, d ; \hat{\mathbf{a}}_{i, m}\right)$ for the first three components $(i=1,2$, and 3 , respectively for component (a), (b), and (c)) and $\sum_{i=1}^{2} \theta_{i}\left(m, d ; \hat{\mathbf{a}}_{i, m}\right)^{2} / \sum_{i=1}^{3} \theta_{i}\left(m, d ; \hat{\mathbf{a}}_{i, m}\right)^{2}$ for the last component, where $\hat{\mathbf{a}}_{i, m}=\left\{\hat{a}_{i, m, 0}, \ldots, \hat{a}_{i, m, 2 K}\right\}$ is the vector of coefficients estimated for each of the three components $(i=1,2,3)$, each delivery month $(m)$, and for each of the four commodities. ${ }^{11}$

\section{(a) Natural gas}

In panel (a) of figure 1, the loadings of the LT factor estimated for natural gas indicate two notable features. First, for all twelve contracts, the estimated factor loadings increase as the contract approaches the maturity date. Second, the factor loadings in the last few months of trading are substantially higher for the contracts maturing in winter than those maturing in summer. The loadings of the ST factor exhibit the same features, but in greater magnitude than those observed for the LT factor (panel b). In addition, for all twelve contracts, the loadings of the ST factor start increasing rapidly in May, before which they are virtually zero for all twelve contracts. In panel (c) of figure 1, the variance of the idiosyncratic error, particularly that for winter contracts, increases very rapidly as the contract approaches maturity. This indicates that high volatility in the last one month of trading, commonly referred as the maturity effect, represents market shocks that are specific to each contact and are of a very short-term nature. Unlike the two common factors, the idiosyncratic errors are not contemporaneously correlated across concurrently traded contracts. Thus, a rapid increase in the variance of the idiosyncratic errors implies that correlation between nearby and distant futures contracts decreases rapidly over the winter season (panel d).

These estimates of the factor loadings and the variance of the idiosyncratic error in the estimated flexible model are consistent with the price dynamics implied by the theory of storage for natural gas. In the flexible model (1), the total variance of log futures price change is given by $\sum_{i=1}^{3} \theta_{i}\left(m, d ; \hat{\mathbf{a}}_{i, m}\right)^{2}$ at $d$ days before the contract maturing in the month $m$. Thus,

\footnotetext{
${ }^{11}$ The denominator in the formula for component (d) represents the total variance, owning to the assumption that two latent factors and idiosyncratic errors are uncorrelated one another.
} 
high factor loadings and high variance of the idiosyncratic errors translate to high volatility of winter contracts in the last few months of trading. During this peak-demand period, tight demand-supply imbalance causes demand or supply shock of even a small magnitude to follow a large price swing, which cannot be absorbed through adjusting inventory because the inventory is effectively zero at the end of the demand season. Low inventory also means that the inter-temporal price linkage breaks at the end of the winter peak-demand season because, in any normal year, no physical stock is carried over from late winter (when price peaks) to early spring (when price is the lowest). The estimated flexible model reflects this feature with a large share of price variation accounted for by the ST factor and the idiosyncratic error for the December through March contracts in their last few months of trading. During the same period, the loadings of the ST factor and the variance of the idiosyncratic error stays very low for contracts maturing in May and thereafter.

[Insert figure 1 somewhere here]

\section{(b) Corn}

In figure 2, the estimated flexible model reveals complex volatility dynamics for corn. The depicted volatility pattern differs from natural gas, yet it is characterized by seasonality in the supply of the underlying commodity. In this figure, the loadings of both the LT and ST factor start increasing for all five contracts around April and peaks in July to August (panels a and b). This observation implies that large price fluctuations of contract prices during this period are highly correlated across the five contracts maturing in the post-harvest season. High price volatility during this period reflects the arrival of important information. In particular, corn crops in the U.S. are typically planted in early April through June and harvested later in the year, usually from September to early November. The date and yield of harvest are determined by weather conditions during summer. Thus, the contracts maturing post-harvest exhibit large price fluctuations and high cross-contract price correlation during this period. Volatility starts decreasing in mid-summer after most weather conditions are revealed and reaches the lowest point when actual harvesting is realized around October. Over these periods, prices move very closely for five contracts maturing post-harvest, because corn is an annual crop and one harvest in the current year needs to be stored for consumption until the new harvest arrives in the subsequent year. In normal years, the current harvest is fully consumed during the demand season and no inventory is carried over to the post-harvest season. Thus, the contracts maturing post-harvest show minimal price movement before 
these crops are planted in early spring. Panel (c) of figure 2 indicates that much of high volatility in the last one month of trading originates in the contract specific errors. The variance of the idiosyncratic error is particularly high for the July contract. This is conveniently explained by low inventory at the end of the demand year, which does not allow unexpected demand and/or supply shocks to be absorbed through inventory adjustment. High variance of contract-specific shock means that a small share of variance is accounted for by the two common factors. This share and, consequently, the correlation among concurrently traded contracts decrease rapidly in the last one month of the trading period, particularly for the July contract (panel d).

[Insert figure 2 somewhere here]

\section{(c) Crude oil}

Crude oil is generally thought to have much weaker demand seasonality than natural gas. However, the estimated flexible model indicates moderate seasonality and maturity effects for the volatility of crude oil price (figure 3). In panel a of figure 3, the estimated loadings of the LT factor is slightly higher for all twelve contracts during winter months. The volatility also increases for all twelve contracts in the last two months of trading, and volatility in this period is slightly higher for winter (January and February) and summer (July and August) contracts. Much of this high volatility in the last two months of trading is captured by the ST factor and the idiosyncratic errors (panels b and c), indicating that this high volatility is caused by shocks that are not persistent and have little impact on the prices of distant maturity contracts. The variance of the idiosyncratic error increases rapidly in the last month of trading and is particularly high for the January and two summer contracts, causing substantial declines in correlation between these contracts and other concurrently traded contracts (panel d).

[Insert figure 3 somewhere here]

\section{(d) Gold}

In figure 4, the estimated flexible model shows virtually no seasonality or maturity effect for gold price volatility. For all six contracts, the estimated loadings of the LT factor are slightly higher from October to March, yet this seasonal difference is negligible, with 
variation ranging no more than 3.5 percent of the average (panel a). Another feature that differentiates gold from the other three commodities is that the estimated loadings of the ST factor and the variance of the idiosyncratic errors are very small for all contracts; they are on average 5.7 percent and 1.7 percent, respectively, of the estimated loadings of the LT factor. These results imply that much of the price shocks to the gold markets are of a long-term nature. High persistence of price shocks is as expected because gold is traded primarily for investment rather than consumption and, unlike other exhaustible resources, the amount of its deposits is well known. Due to large loadings of the LT factor, relative to the variance of the idiosyncratic error, the two common factors account for a large share of the total variance, resulting in high cross-contract correlation for gold as seen in panel (d) of figure 4 .

[Insert figure 4 somewhere here]

\subsection{Estimation results: Conventional two-factor model}

Figures 5 through 8 present the results of estimating the conventional two-factor model in the first difference form (8) for the four commodities. These figures plot, for each contract: (a) the variance of futures price attributable to the two common factors, which is calculated as $\hat{\sigma}_{x}^{2}+\hat{\sigma}_{z}^{2} e^{-2 \hat{\kappa} \tau}+\hat{\rho} \hat{\sigma}_{x} \hat{\sigma}_{z} e^{-\hat{\kappa} \tau}$, (b) the variance of the idiosyncratic error $\hat{\sigma}_{u, m}^{2}$, and (c) the share of the variance accounted for by the two common factors as calculated by $1-\hat{\sigma}_{u, m}^{2}\left(\hat{\sigma}_{x}^{2}+\hat{\sigma}_{z}^{2} e^{-2 \hat{\kappa} \tau}+\hat{\rho} \hat{\sigma}_{x} \hat{\sigma}_{z} e^{-\hat{\kappa} \tau}+\hat{\sigma}_{u, m}^{2}\right)^{-1}$. In panel a of figures 5 through 8 , the variance attributable to the two common factors increases exponentially as the contract approaches maturity for all four commodities. This property, as discussed in Section 2, is the direct result of the model specification that the LT and ST factors follow the GBM and MR processes, respectively.

\section{[Insert figure 5 somewhere here]}

The conventional model allows the variance of the idiosyncratic error to vary by contract delivery date but not by time-to-maturity. In panel b of figure 5, the model estimated for natural gas indicates a higher variance of the idiosyncratic error for winter (January through March) contracts than for the other contracts. However, the estimated variance of the idiosyncratic error, even for these winter contracts, is negligible in size, when compared to the variance attributable to the two common factors. These estimates cause the model to 
imply very high cross-contract correlation, which increases as contracts approach maturity because the variance attributable to the ST factor increases exponentially. These implications for the magnitude of cross-contract correlation and its dynamics over time-to-maturity are exactly opposite to the implications of the flexible model.

These results signify the severity of the restrictions imposed by the specifications of the conventional two-factor model. Of the three stochastic components in the right-hand side of the model in (8), the ST factor is the only component that captures the dynamics of the price variance over the trading horizon, yet it only allows the variance to decrease exponentially with time-to-maturity at an identical rate for all contracts. It neither permits non-monotonic change of the price variance nor allows the variance to change at different rates across contracts. The model also restricts the idiosyncratic error, $\Delta u_{T, t}$, to be the only component to capture the cross-contract difference in price variance. The estimation results described above are determined by these specifications of the model. For natural gas, the model allocates a large share of the price variance to the ST factor to capture strong maturity effects. It implies low variance of the idiosyncratic errors because seasonal variation in the volatility of natural gas price is small relative to the maturity effect. The severity of these restrictions is apparent when the model is compared with the flexible model, in which the three components are equally flexible in their seasonal and cross-sectional variation and the composition of the observed price movements among the three components is determined by cross-contract price correlation.

In figures 6 through 8 , the conventional two-factor model estimated for corn, crude oil, and gold exhibits the same results as for natural gas. For all three commodities, the variance attributable to the two common factors decreases gradually with time-to-maturity at the identical rate for all contracts. The variance of the idiosyncratic error exhibits cross-contract variation, with variance slightly higher for the July corn contract, the August gold contract, and the winter crude oil contracts. However, for all three commodities and for all contracts, the variance of the idiosyncratic error is constant over the trading horizon and much smaller than the variance attributable to the two common factors. The results imply that the two common factors account for a large share of price variance and that this share increases as the contract approaches maturity; the implications are exactly opposite to the estimated flexible models. For gold only, the difference between the flexible model and the conventional twofactor model is small because very weak seasonality and maturity effects lead both models to assign a dominant share of the price variance to the LT factor, resulting in very small loadings of the ST factor and a small variance of the idiosyncratic error. 
[Insert figures 6-8 somewhere here]

\subsection{Estimation results: Composite model}

Figures 9 through 12 show the results from estimating the composite model (9). ${ }^{12}$ In figure 9, the variance attributable to the two common factors for natural gas exhibits the same dynamic pattern but is slightly smaller in magnitude than the estimate in the conventional two-factor model (panel a). In panel (b), the variance of the idiosyncratic error estimated for the composite model is substantially greater than the estimates for the conventional twofactor model and exhibits strong seasonality and maturity effect. Strong seasonality and maturity effect of the idiosyncratic error is reflected in the total variance, resulting in a dynamics similar to the one estimated for the flexible model. Nonetheless, the share of the total variance accounted for by the two common factors exhibits different dynamics between the two models simply because the compositions of the price variance among the three components differ between the two models (see figure 9.c and figure 1.d).

[Insert figure 9 somewhere here]

Figures 10 through 12 show that the composite model estimated for corn, crude oil, and gold yields results similar to those for natural gas. The variance attributable to the two common factors decreases with time-to-maturity yet at a slower rate than in the conventional two-factor model. On the other hand, the variance of the idiosyncratic error is estimated substantially greater for the composite model than for the conventional two-factor model. The variance estimate for the composite model also exhibits strong maturity effects with variance in the last month of trading being particularly large for the July contract for corn and the January, July, and August contracts for crude oil. The maturity effect captured by the idiosyncratic error dominates the increase in the loadings of the ST factor, implying that cross-contract correlation decreases rapidly in the last few months of trading. The increase in the variance of the idiosyncratic error near the maturity date is much smaller for gold, resulting in only a marginal reduction in the cross-contract correlation near the maturity dates.

\footnotetext{
${ }^{12}$ In these figures, the variance of futures price attributable to the two common factors is calculated in the same way as for the conventional two-factor model whereas the variance attributable to the idiosyncratic error is given as $\theta_{3}^{2}\left(m, d ; \hat{\mathbf{a}}_{3, m}\right)$.
} 
[Insert figures 10-12 somewhere here]

In summary, estimates of the flexible model of futures returns reveal that the volatility of the three commodity futures; natural gas, corn, and crude oil, exhibit strong seasonality and maturity effects. For natural gas, the volatility in the last few months of trading is particularly high in winter months when demand peaks and inventory is low. Much of the high volatility during this period is captured by the ST factor and the idiosyncratic error because low inventory breaks the inter-temporal price link, resulting in low cross-contract price correlation. For corn, volatility increases in spring through early summer when crops are planted and as much of the weather shocks affecting the growth of these crops are revealed. During this period, contracts maturing in the post-harvest season exhibit high price correlation. In the same period, the July contract that matures before harvest is subject to high contract-specific shock because low inventory at the end of the demand season breaks the inter-temporal price link. Crude oil exhibits high price volatility both in winter and summer months, but the seasonality is moderate relative to natural gas and corn.

The conventional two-factor model fails to capture these complex volatility dynamics of the three consumption commodities because of strong restrictions imposed on the factor loadings and the variance of the idiosyncratic error. In particular, the model allows neither a cross-contract nor a seasonal variation in the factor loadings. Neither does it allow a variation in the variance of the idiosyncratic error by time-to-maturity. Consequently, the model captures cross-contract variation in the price volatility solely by the idiosyncratic error and the maturity effect by the ST factor only. Flexible specification of the variance of the idiosyncratic error permits the composite model to alleviate, albeit imperfectly, the restrictions imposed in the conventional two-factor model. The model replicates reasonably well the complex volatility dynamics of the consumption commodities, yet it implies the dynamics of cross-contract correlations that are substantially different from the pattern implied by the flexible model.

\section{Implications for Optimal Hedging Strategy}

In the previous section, estimates of the factor loadings and the variance of the idiosyncratic errors differ substantially across three models. This section compares the three models by their implications for an optimal hedging strategy. 


\subsection{Optimal hedging}

I consider a trader with a spot position, $Q$, in period $t$, who simultaneously takes a short position in $X$ futures contracts for delivery at $\tau>t$. At $t+k<\tau$, the trader clears its position by selling $Q$ units in the spot market and buying $X$ futures contracts for delivery at $\tau$.

Returns to this trader's portfolio from $t$ to $t+k$ is

$$
W_{t+k}=\Delta \ln S_{t+k} Q-\Delta \ln F_{\tau, t+k} X=\left(\Delta \ln S_{t+k}-\eta \Delta \ln F_{\tau, t+k}\right) Q
$$

where $\Delta \ln S_{t+k}$ and $\Delta \ln F_{\tau, t+k}$ are, respectively, the change from $t$ to $t+k$ of the log spot price and $\log$ price of futures contract for delivery at $\tau$ and $\eta=X Q^{-1}$ is the hedge ratio. The variance of portfolio return is

$$
\mathrm{V}\left[W_{t+k}\right]=Q^{2}\left(\mathrm{~V}\left[\Delta \ln S_{t+k}\right]+\eta^{2} \mathrm{~V}\left[\Delta \ln F_{\tau, t+k}\right]-2 \eta \operatorname{cov}\left[\Delta \ln S_{t+k}, \Delta \ln F_{\tau, t+k}\right]\right)
$$

which is minimized when the hedge ratio is set as

$$
\eta^{*}=\frac{\operatorname{cov}\left[\Delta \ln S_{t+k}, \Delta \ln F_{\tau, t+k}\right]}{\mathrm{V}\left[\Delta \ln F_{\tau, t+k}\right]}
$$

The minimum variance attained by this hedge ratio is

$$
\mathrm{V}\left[W_{t+k} \mid \eta^{*}\right]=\mathrm{V}\left[\Delta \ln S_{t+k}\right]\left(1-\rho_{\tau, t+k}^{2}\right) Q^{2}
$$

where $\rho_{\tau, t+k}$ is the correlation between the change in the log spot price and the change in log price of the futures contract for delivery at $\tau$ over the period between $t$ and $t+k$.

In the above variance minimization problem, the delivery date $\tau$ of the futures contract included into the portfolio is taken as exogenous. In many organized exchanges, however, multiple contracts with different maturity dates are traded simultaneously. Thus, the hedger can choose from these multiple contracts one that attains the minimum portfolio variance. Equation (11) indicates that, given the time of entry $(t)$ and hedging horizon $(k)$, the portfolio variance is minimized when it includes the contract that exhibits the highest correlation with the spot price over the hedging horizon. Once this contract is identified, the optimal hedge 
ratio is determined according to (10) as the ratio of the covariance between the spot and futures returns to the variance of futures returns.

\subsection{Optimal hedging strategy according to the three models}

It is apparent in (10) and (11) that specifications of variance and covariance dynamics of spot and futures prices play an important role in determining the optimal hedging strategy. Given the time of entry $(t)$ and hedging horizon $(k)$, one can calculate the optimal hedge ratio $(\eta)$ and the associated portfolio variance for every possible choice of futures contract included into the portfolio $(\tau)$, based on the three models of daily futures returns estimated in Section 3. In particular, the variance of log futures returns in the denominator of the expression (10) is calculated as

$$
\mathrm{V}\left[\Delta \ln F_{\tau, t}\right]= \begin{cases}\hat{\sigma}_{x}^{2}+\hat{\sigma}_{z}^{2} e^{-2 \hat{\kappa} d}+2 \hat{\rho} \hat{\sigma}_{x} \hat{\sigma}_{z} e^{-\hat{\kappa} d}+\hat{\sigma}_{\tau}^{2} & \text { for conventional two-factor model } \\ \theta_{1}^{2}\left(\tau, d ; \hat{\mathbf{a}}_{1}\right)+\theta_{2}^{2}\left(\tau, d ; \hat{\mathbf{a}}_{2}\right)+\theta_{3}^{2}\left(\tau, d ; \hat{\mathbf{a}}_{3}\right) & \text { for flexible model } \\ \hat{\sigma}_{x}^{2}+\hat{\sigma}_{z}^{2} e^{-2 \hat{\kappa} d}+2 \hat{\rho} \hat{\sigma}_{x} \hat{\sigma}_{z} e^{-\hat{\kappa} d}+\theta_{3}^{2}\left(\tau, d ; \hat{\mathbf{a}}_{3}\right) & \text { for composite model }\end{cases}
$$

where $d=\tau-t$ is the time to delivery of the contract maturing at $\tau$. Similarly, using the nearby futures price as the proxy for the spot price, ${ }^{13}$ one can calculate the covariance between the log returns to spot (nearby futures) and futures contract for delivery at $\tau$ as

$$
\operatorname{cov}\left[\Delta \ln S_{t}, \Delta \ln F_{\tau, t}\right]=\left\{\begin{array}{lr}
\hat{\sigma}_{x}^{2}+\hat{\sigma}_{z}^{2} e^{-\hat{\kappa}\left(d_{0}+d\right)}+\hat{\rho} \hat{\sigma}_{x} \hat{\sigma}_{z}\left(e^{-\hat{k} d_{0}}+e^{-\hat{\kappa} d}\right) & \text { for conventional two-factor } \\
\theta_{1}\left(\tau_{0}, d_{0} ; \hat{\mathbf{a}}_{1}\right) \cdot \theta_{1}\left(\tau, d ; \hat{\mathbf{a}}_{1}\right) & \text { and composite model } \\
+\theta_{2}\left(\tau_{0}, d_{0} ; \hat{\mathbf{a}}_{2}\right) \cdot \theta_{2}\left(\tau, d ; \hat{\mathbf{a}}_{2}\right) & \text { for flexible model }
\end{array}\right.
$$

where $d_{0}=\tau_{0}-t$ and $\tau_{0}$ is the delivery date of the nearby contract.

The first expression in (13) indicates that, for the conventional two-factor model and the composite model, the covariance between the log spot and log futures returns declines monotonically with time-to-maturity $(d)$ of the futures contract, which results from the specification that the two common factors follow the GBM and MR processes in (4). Furthermore, for the conventional two-factor model, the variance of log futures returns also declines monotonically with time-to-maturity in (12) yet at a slower pace than the decline in the covariance of the spot and futures returns. Thus, the correlation between the two prices

${ }^{13}$ This approximation is justified by the standard arbitrage argument that the price of the futures contract converges to the spot price as the contract approaches the maturity date (see, for example, Hull, 2002). 
declines monotonically with time-to-maturity and the model proposes that the hedger should include into the portfolio the contract that is the closest to maturity to minimize the portfolio variance. This implication for the optimal hedging strategy generally applies to the composite model except that the model suggests the use of a more distant contract when the close-tomaturity contracts are increasingly subject to high idiosyncratic variance $\theta_{3}\left(\tau, d ; \hat{\mathbf{a}}_{3}\right)$ (in which case, the variance of the futures contract increases faster than the covariance between the two prices). For the flexible model, the estimated factor loadings $\left(\theta_{1}\left(\tau, d ; \hat{\mathbf{a}}_{1}\right)\right.$ and $\left.\theta_{2}\left(\tau, d ; \hat{\mathbf{a}}_{2}\right)\right)$ and the standard deviation of the idiosyncratic error $\left(\theta_{3}\left(\tau, d ; \hat{\mathbf{a}}_{3}\right)\right)$ exhibit substantial seasonal and cross-contract variations. Because the resulting spot-futures correlations also exhibit seasonal and cross-sectional variations, the model proposes potentially very complex hedging strategies that require both the size $(\eta)$ and position $(\tau)$ in the futures market to be adjusted frequently across seasons.

Using the variance and covariance implied by each of the three models, I calculate the optimal hedge ratio for a one-day hedging horizon $(k=1)$ and $t$ ranging from the first day to the last day of a calendar year. ${ }^{14}$ For each $t$, I compare among the futures contracts maturing in the subsequent twelve months by their correlation with the spot price and calculate the optimal hedge ratio for the portfolio including the contract that exhibits the highest correlation with the spot price.

Figure 13 illustrates, for each of the four commodities and for each of the three models, how the futures contract included into the optimal portfolio shifts by the date of entry. The figure shows that the optimal contract differs substantially among the three models. In the figure, the optimal hedging strategy based on the conventional two-factor model includes the second position contract which is the closest to maturity after the nearby contract. This result is expected because the model stipulates that, for each contract, the share of the variance accounted for by the two common factors increases as the contract approaches its maturity date. Thus, on any particular day, the contract closer to maturity exhibits a higher correlation with the spot price unless it receives an exceptionally large idiosyncratic error. The exception is observed for corn in July through mid-August, during which the nearby (September)

\footnotetext{
${ }^{14}$ I consider a hedging horizon of a single day because, in the absence of transaction cost for adjusting futures position, the best (variance-minimizing) strategy for a longer hedging horizon is to alter the choice of contract $(\tau)$ and the hedge ratio $(\eta)$ every day so as to follow the optimal strategy for a one-day horizon. Staying with the previous-day position could be optimal in the presence of transaction cost. The best strategy in such a setting will depend on various factors such as the length of the hedging horizon, the size of the transaction cost, and the risk aversion coefficient of the hedger. This is beyond the scope of the paper and hence is left for future research.
} 
contract exhibits higher correlation with the third position (March) contract than with the second position (December) contract because of the high idiosyncratic variance of the latter.

\section{[Insert figure 13 somewhere here]}

The flexible model and composite model also suggest the use of the near-to-maturity (second position) contract to hedge against the spot price risk for gold and corn. For crude oil and natural gas, the two models suggest the use of a more distant contract; typically the third position contract for crude oil and the third or fourth position contract for natural gas. The two models suggest the use of distant contracts because the contracts closer to maturity are subject to high idiosyncratic shocks and hence exhibit low correlation with the nearby contract. For natural gas, the optimal portfolio includes the June or July contract early in the calendar year (February to May). During this time, contracts for earlier maturity are subject to high idiosyncratic shocks because low inventory at the end of the demand year disconnects the inter-temporal price links. The optimal contract shifts gradually from June to mid-August and, after that, it switches to a winter (December or January) contract. In this post summer season, winter contracts are subject to little idiosyncratic shocks and much of their price movements reflect common market shocks. Around the beginning of November, winter contracts start receiving idiosyncratic shocks and the optimal contract is replaced with a more distant contract.

Figure 14 plots the optimal hedge ratio against the time of entry for each of four commodities. In panel (c), the optimal hedge ratio for gold is close to one throughout the year for all three models. For gold, the variance of the idiosyncratic error is very small and a large share of the price variance is accounted for by the LT factor in all three models. Thus, crosscontract correlation is very high and the variance and covariance in expression (10) are about the same magnitude, resulting in the optimal hedge ratio of close to one. Similarly, the optimal hedge ratio for corn remains close to one in panel (d), yet it is slightly lower for the flexible model than for the other two models because the model implies higher idiosyncratic error (and hence the lower covariance of the two prices) than the other two models.

\section{[Insert figure 14 somewhere here]}

For the other two commodities, the optimal hedge ratio differs substantially across the three models. For both natural gas and crude oil, the optimal hedge ratio suggested by the 
conventional two-factor model shows little seasonal variation while it increases gradually towards the end of each calendar month. The model stipulates that the variance attributable to the two common factors increases exponentially as the contract approaches its maturity date. The specification follows that the covariance between spot and futures return increases faster than the variance of futures return, resulting in a gradual increase in the optimal hedge ratio.

The composite model implies a similar strategy as the conventional two-factor model for hedging crude oil price risk, except that it suggests a much smaller variation of the optimal hedge ratio within each month. In contrast, the strategy based on the flexible model indicates a large increase in the optimal hedge ratio within each month simply because the estimated factor loadings increase much faster in this model than in the other two models. For natural gas, the optimal hedge ratio suggested by the flexible and composite model exhibits substantial seasonal variation, reflecting the seasonal variation in the position of the futures contract included in the optimal portfolio. In general, the optimal hedge ratio is high when the portfolio includes a distant contract, because a distant contract is subject to little idiosyncratic volatility and hence results in a low variance of futures return in the denominator of (10). An optimal hedge ratio substantially above unity is often suggested because the futures contract included in the portfolio exhibits small variation, a dominant share of which represents common market shocks.

Table 2 presents the sum of the portfolio variance over a one-year hedging horizon expressed as the ratio to the variance of $\log$ spot price without hedging. ${ }^{15}$ In the table, all three hedging strategies reduce portfolio variance dramatically. The three strategies are particularly effective in reducing spot price risk for gold, for which cross-contract correlation is particularly high throughout the year. Portfolio variance is relatively high for crude oil and natural gas, ranging from around 18 to 26 percent of the variance of the spot price. Hedging

\footnotetext{
${ }^{15}$ These numbers are calculated under the assumption that the futures returns follow the process as specified in the estimated flexible model, which receives the strongest empirical support for all four commodities. Specifically, the variance of optimal portfolio is calculated, for each of the three hedging strategies and for each entry date $(t)$, as $\mathrm{V}\left[W_{t+1}(t)\right]=\mathrm{V}\left[\Delta \ln S_{t+1}\right]+\eta^{*}(t)^{2} \mathrm{~V}\left[\Delta \ln F_{\tau^{*}(t), t+1}\right]-2 \eta^{*}(t) \operatorname{cov}\left[\Delta \ln S_{t+1}, \Delta \ln F_{\tau^{*}(t), t+1}\right]$ where $\tau^{*}(t)$ and $\eta^{*}(t)$ are the delivery month of the futures contract included into the portfolio and the hedge ratio according to the optimal strategy as seen in figures 13 and 14, respectively, and the variance and covariance are evaluated according to the estimated flexible model, i.e., $\mathrm{V}\left[\Delta \ln S_{t+1}\right]=\sum_{i=1}^{3} \theta_{i}^{2}\left(\tau_{0}, d_{0} ; \hat{\mathbf{a}}_{i}\right)$, $\mathrm{V}\left[\Delta \ln F_{\tau^{*}(t), t+1}\right]=\sum_{i=1}^{3} \theta_{i}^{2}\left(\tau^{*}(t), d^{*}(t) ; \hat{\mathbf{a}}_{i}\right), \quad$ and $\operatorname{cov}\left[\Delta \ln S_{t+1}, \Delta \ln F_{\tau^{*}(t), t+1}\right]=\sum_{i=1}^{2} \theta_{i}\left(\tau_{0}, d_{0} ; \hat{\mathbf{a}}_{i}\right) \cdot \theta_{i}\left(\tau^{*}(t), d^{*}(t) ; \hat{\mathbf{a}}_{i}\right)$ where $d^{*}(t)=\tau^{*}(t)-t$.
} 
strategies are less effective for these two commodities because the nearby contract is subject to a large contract-specific shock near maturity.

For all four commodities, portfolio variance is the smallest for a strategy based on the flexible model. The composite model performs better than the conventional model in hedging spot price risks for crude oil and natural gas. However, the strategy results in a substantially greater portfolio variance than the strategy based on the flexible model for natural gas. This result signifies the importance of specifying factor loadings by a flexible functional form to properly account for not only seasonal and temporal variation in the price variance but also the variation in the cross-contract correlation, the latter of which plays a significant role in designing an effective hedging strategy.

\section{Conclusion}

In this study, I compare a conventional two-factor term-structure model of commodity futures with an alternative modeling approach of specifying variance of daily futures returns directly by flexible functions. Empirical estimation of the flexible model of futures returns with daily futures price data from four commodity markets reveal that the price volatility exhibits strong maturity effects for three consumption commodities (crude oil, natural gas and corn) and significant seasonal variation for commodities with strong seasonality in demand (natural gas) or supply (corn). The futures price volatility for three consumption commodities also exhibits complex dynamics in its composition among the two common factors and the contract-specific shock; volatility increases rapidly in the last month of trading, a large share of which emanates from the contract-specific shock. Consequently, the correlation between nearby futures price and prices of more distant contracts declines sharply as the contract approaches its maturity date. The model also reveals that the inter-temporal price linkage breaks at the end of winter for natural gas and at around September to early November for corn. The finding supports the implication of the theory of storage that the inter-temporal price link breaks when inventory clears out, which happens at the end of winter peak-demand season for natural gas and in September right before the new harvest arrives for corn.

The conventional two-factor Gaussian model cannot replicate these complex price dynamics due to restrictive specifications stipulated for the stochastic processes of the underlying factors and the variance of the measurement error. In particular, the commonly considered model specification forces the short-term factor to capture entirely the variation in 
the futures price volatility by time-to-maturity while it assigns the measurement error to capture the seasonal and cross-contract variation in the futures price volatility. The specification also stipulates that the variance attributable to the two common factors decreases exponentially with time-to-maturity at an identical rate for all contracts. Due to these restrictions, the model implies that cross-contract correlation increases monotonically as the contract approaches maturity, which is exactly opposite to the implication of the flexible model.

The composite model, allowing a flexible variance structure of the idiosyncratic error, performs reasonably well in replicating the complex price dynamics depicted by the flexible model. The results highlight that specifying a flexible variance structure of the idiosyncratic error alone can improve the ability of conventional term-structure models to replicate the complex volatility dynamics of consumption commodities. These findings caution against a recent trend in the development of term-structure models, which focuses exclusively on adopting more flexible stochastic processes of common stochastic factors while maintaining restrictive structures on the variance of the measurement error.

An incorrect portrayal of volatility and cross-contract correlation can lead the conventional term-structure model to suggest hedging strategies that are less effective than the strategy based on the flexible model. The strategy based on the composite model is also less effective than the flexible model, particularly for the two consumption goods with strong seasonality in demand or supply. These results indicate that specifying the variance of the idiosyncratic error alone cannot replicate properly the complex dynamics of the price correlation across concurrently traded contracts, which is critical for designing an effective hedging strategy and for proper pricing of derivative contracts whose payoff depends on the realization of price spreads. 


\section{References}

Black, F., and Scholes, M. 1973. The pricing of options and corporate liabilities. Journal of Political Economy, 81: 637-654.

Cortazar, G., and Schwartz, E.S. 1994. The valuation of commodity-contingent claims, Journal of Derivatives, 1: 27-39.

Engle, R.F., and Kroner, K.F. 1985. Multivariate simultaneous generalized ARCH. Econometric Theory, 11: 122-150.

Gibson, R., and Schwartz, E.S. 1990. Stochastic convenience yield and the pricing of oil contingent claims. Journal of Finance, 45: 959-976.

Hamilton, J. D. 1994. State-space models. In R. F. Engle, D. L. McFadden eds., Handbook of Econometrics (Vol. 4). Amsterdam: Elsevier.

Heath, D., Jarrow, R., and Morton, A. 1992. Bond pricing and the term structure of interest rates: A new methodology for contingent claims valuation, Econometrica, 60(1): 77-105.

Hull, J.C. 2002. Options, Futures, and Other Derivatives. 5th ed. Prentice Hall.

Lautier, D. 2005. Term structure models of commodity prices: A review. Journal of Alternative Investments, 8(1): 42-64.

Lucia, J., and Schwartz, E.S. 2002. Electricity prices and power derivatives: Evidence from the Nordic power exchange. Review of Derivatives Research, 5: 5-50.

Manoliu, M., and Tompaidis, S. 2002. Energy futures prices: Term structure models with Kalman filter estimation. Applied Mathematical Finance, 9: 21-43.

Nielsen, M.J., and Schwartz, E.S. 2004. Theory of storage and the pricing of commodity claims. Review of Derivatives Research, 7: 5-24.

Routledge, B.R., Seppi, D.J., and Spatt, C.S. 2000. Equilibrium forward curves for commodities, Journal of Finance, 55: 1297-1338.

Schwartz, E.S. 1997. The Stochastic behavior of commodity prices: Implications for valuation and hedging. Journal of Finance, 52: 923-973.

Schwartz, E.S., and Smith, J.E. 2000. Short-term variations and long-term dynamics in commodity prices. Management Science, 46: 893-911.

Smith, A. 2005. Partially overlapping time series: A new model for volatility dynamics for commodity futures. Journal of Applied Econometrics, 20: 405-422.

Sorensen, C. 2002. Modeling seasonality in agricultural commodity futures. Journal of Futures Markets, 22(5): 393-426.

Suenaga, H., and Smith, A. 2011. Volatility dynamics and seasonality in energy prices: Implications for crack-spread price risk. Energy Journal, 32(3): 27-58.

Williams, J.C., and Wright, B.D. 1991. Storage and Commodity Markets. Cambridge University Press, New York. 


\section{Acknowledgement}

I gratefully acknowledge insightful comments from two anonymous reviewers, which helped improve the paper substantially. This research was conducted with the financial support from the Australian Research Council Discovery Grant (DP1094499). The author benefitted from discussions with Aaron Smith, Felix Chan, Harry Bloch, and participants of the MODSIM 2011. 


\section{Appendix - Table and Figures}

Table 1. Model Selection Test

\begin{tabular}{|c|c|c|c|c|}
\hline & \multicolumn{4}{|c|}{ Commodity } \\
\hline & Corn & Crude Oil & Gold & Natural Gas \\
\hline \multicolumn{5}{|l|}{ Sample size } \\
\hline All & 48762 & 70800 & 43820 & 52780 \\
\hline \multicolumn{5}{|l|}{ By contract delivery month } \\
\hline Jan & & 5970 & & 4408 \\
\hline Feb & & 5882 & 7255 & 4411 \\
\hline Mar & 9988 & 5923 & & 4406 \\
\hline Apr & & 5904 & 7296 & 4383 \\
\hline May & 9517 & 5864 & & 4369 \\
\hline June & & 5853 & 7312 & 4391 \\
\hline July & 9764 & 5811 & & 4400 \\
\hline Aug & & 5803 & 7328 & 4402 \\
\hline Sep & 9255 & 5887 & & 4403 \\
\hline Oct & & 5919 & 7334 & 4402 \\
\hline Nov & & 5933 & & 4404 \\
\hline Dec & 10238 & 6051 & 7295 & 4401 \\
\hline \multicolumn{5}{|c|}{ By days to maturity (business days before the first day of delivery month) } \\
\hline$-21-0$ & 1728 & & 3057 & \\
\hline $1-42$ & 5665 & 10421 & 6800 & 8238 \\
\hline $43-84$ & 5665 & 12463 & 6797 & 8930 \\
\hline $85-126$ & 5664 & 12460 & 6794 & 8925 \\
\hline $127-168$ & 5665 & 12270 & 6795 & 8926 \\
\hline $169-210$ & 5664 & 12025 & 6791 & 8920 \\
\hline $211-252$ & 5662 & 11161 & 6786 & 8841 \\
\hline $253-294$ & 5570 & & & \\
\hline $295-336$ & 4756 & & & \\
\hline $337-378$ & 2723 & & & \\
\hline Number of contracts & 142 & 307 & 168 & 223 \\
\hline Number of trading days & 6808 & 6204 & 6779 & 4441 \\
\hline \multicolumn{5}{|c|}{ Number of parameters estimated } \\
\hline Flexible & 124 & 285 & 147 & 285 \\
\hline Conventional two factor & 11 & 18 & 12 & 18 \\
\hline Composite & 46 & 102 & 54 & 102 \\
\hline \multicolumn{5}{|l|}{ Akaike Information Criterion } \\
\hline Flexible & -428928 & -697588 & -558094 & -431278 \\
\hline Conventional two factor & -400078 & -618955 & -525549 & -393527 \\
\hline Composite & -416075 & -691672 & -551946 & -421183 \\
\hline \multicolumn{5}{|c|}{ Schwarz Information Criterion } \\
\hline Flexible & -427837 & -694975 & -556817 & -428749 \\
\hline Conventional two factor & -399981 & -618790 & -525444 & -393367 \\
\hline Composite & -415670 & -690737 & -551477 & -420277 \\
\hline
\end{tabular}

Table 2. Theoretical Valuation of Hedging Performance

\begin{tabular}{lrrr}
\hline & Conventional & Flexible & \multicolumn{2}{c}{ Composite } \\
\hline Crude oil & $20.94 \%$ & $18.54 \%$ & $18.91 \%$ \\
Natural gas & $26.18 \%$ & $20.09 \%$ & $25.54 \%$ \\
Gold & $0.14 \%$ & $0.14 \%$ & $0.17 \%$ \\
Corn & $8.78 \%$ & $7.97 \%$ & $9.49 \%$ \\
\hline
\end{tabular}


Figure 1. Factor loadings, variance of idiosyncratic error, and share of total variance accounted for by two common factors in flexible model: Natural Gas

(a) Factor loading 1

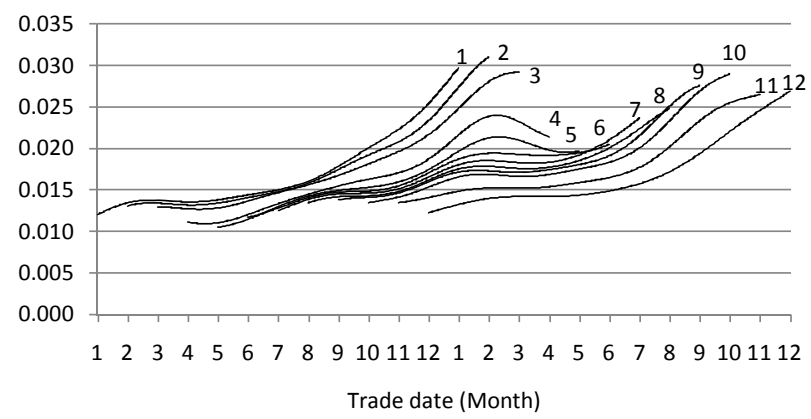

(b) Factor loading 2

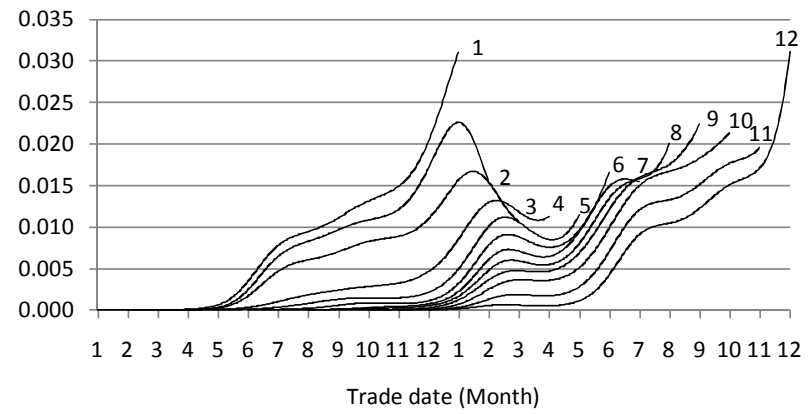

(c) Standard deviation of idiosyncratic error

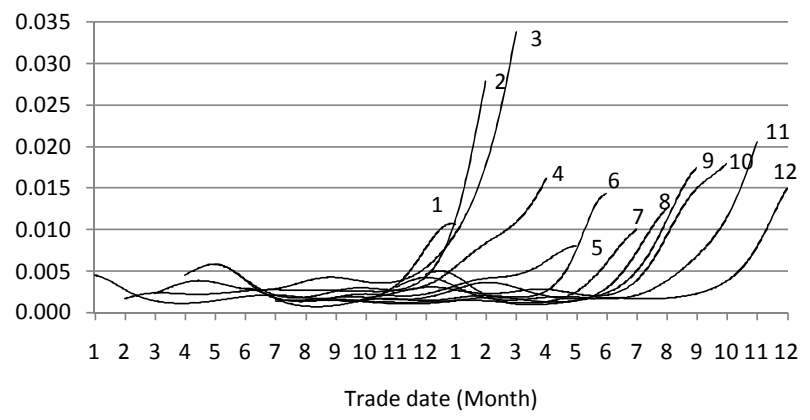

(d) Share of total variance accounted for by two common factors

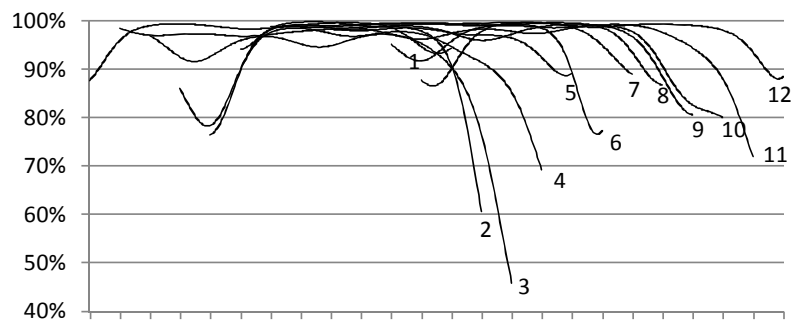

1233455677891011121223445667889101112

Trade date (Month) 
Figure 2. Factor loadings, variance of idiosyncratic error, and share of total variance accounted for by two common factors in flexible model: Corn

(a) Factor loading 1

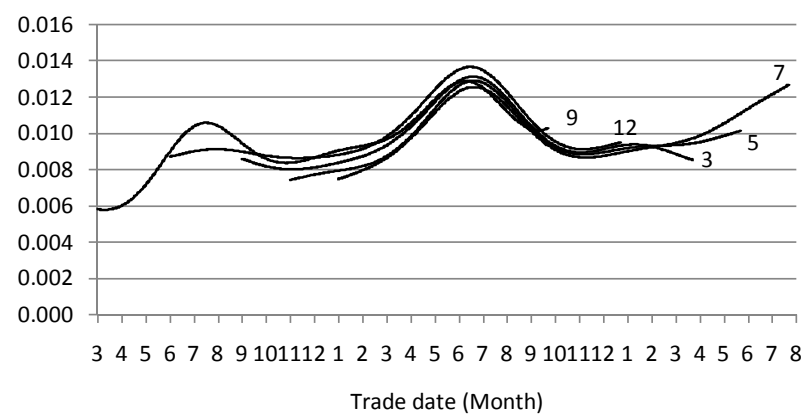

(b) Factor loading 2

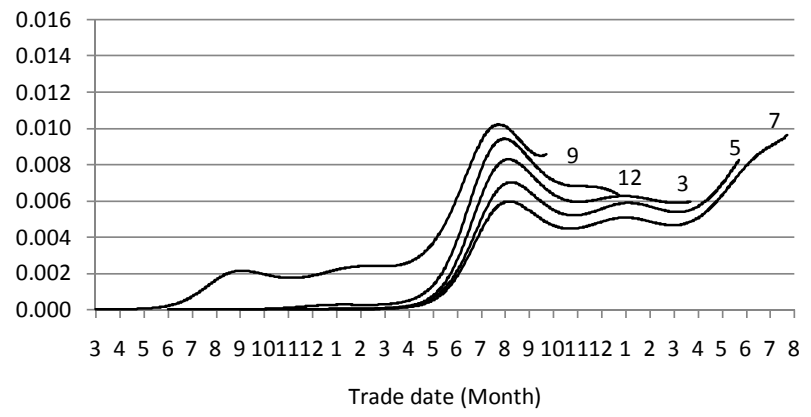

(c) Standard deviation of idiosyncratic error

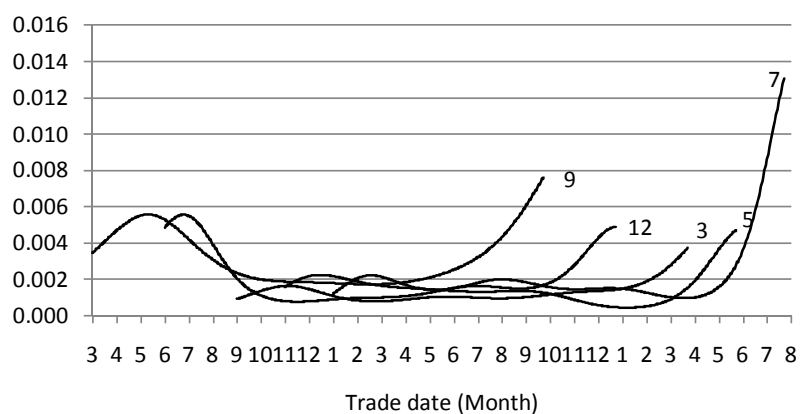

(d) Share of total variance accounted for by two common factors

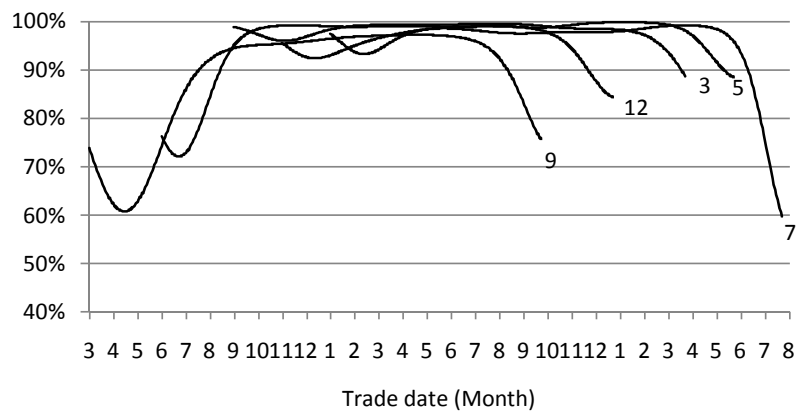


Figure 3. Factor loadings, variance of idiosyncratic error, and share of total variance accounted for by two common factors in flexible model: Crude oi

(a) Factor loading 1

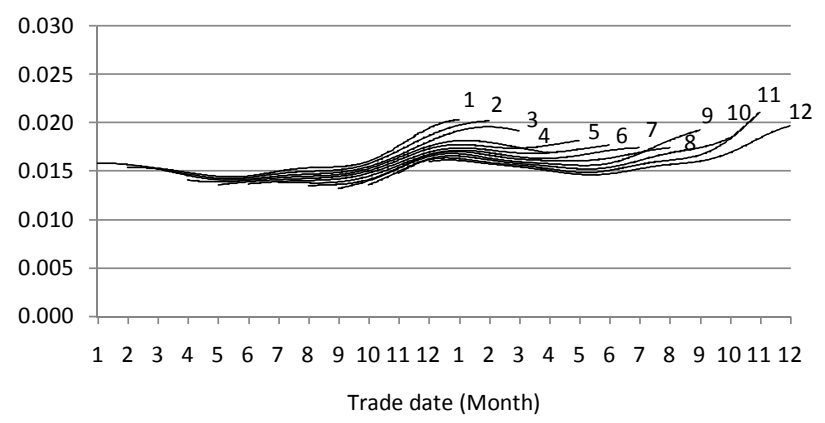

(b) Factor loading 2

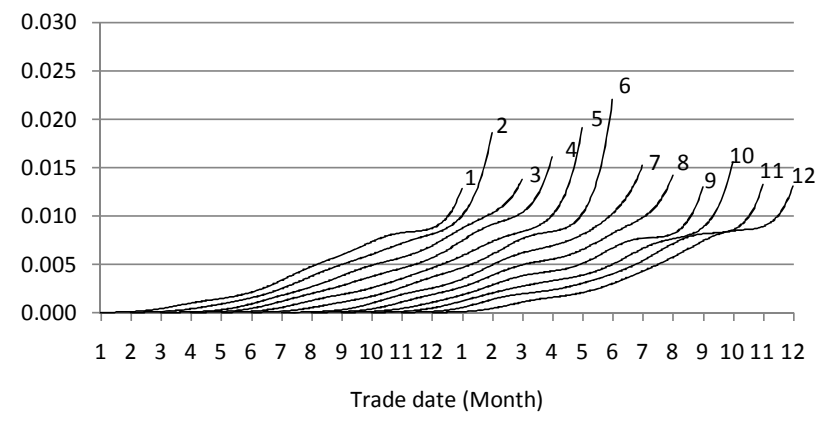

(c) Standard deviation of idiosyncratic error

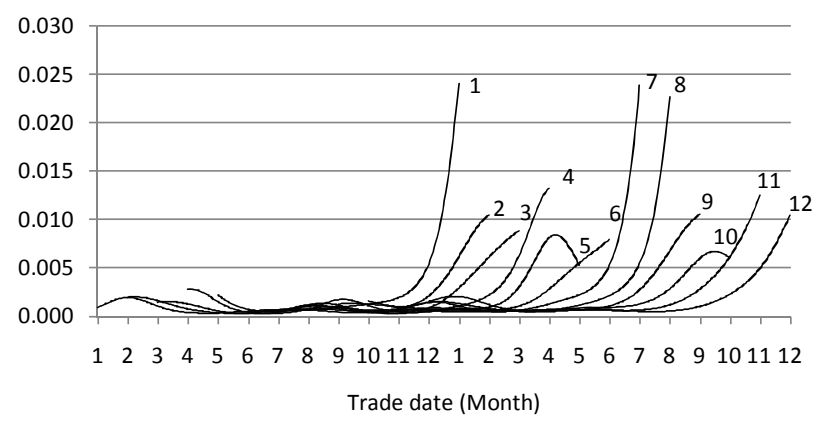

(d) Share of total variance accounted for by two common factors

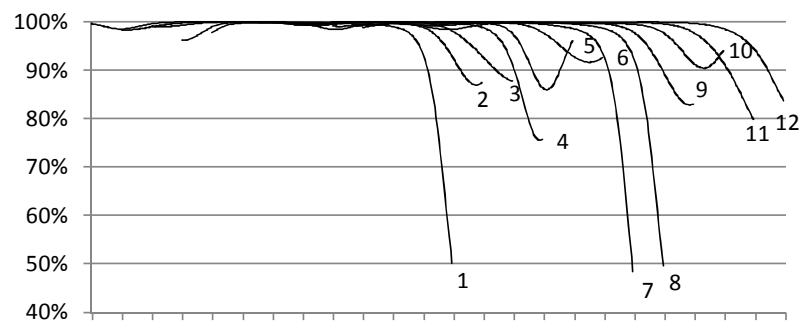

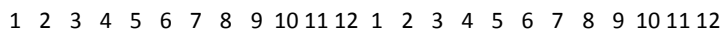

Trade date (Month) 
Figure 4. Factor loadings, variance of idiosyncratic error, and share of total variance accounted for by two common factors in flexible model: Gold

(a) Factor loading 1

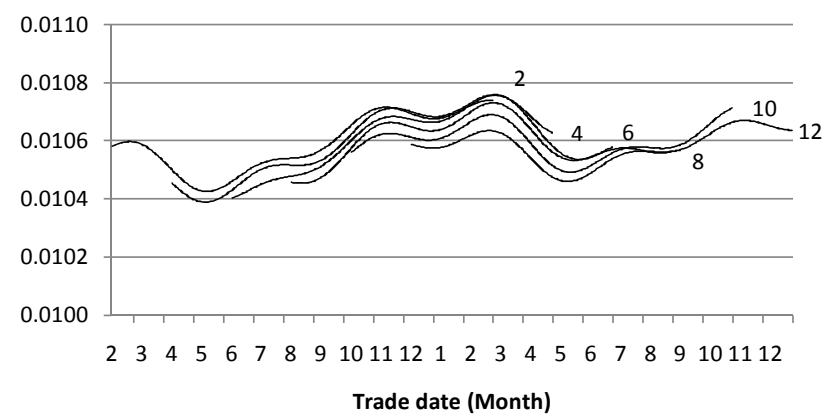

(b) Factor loading 2

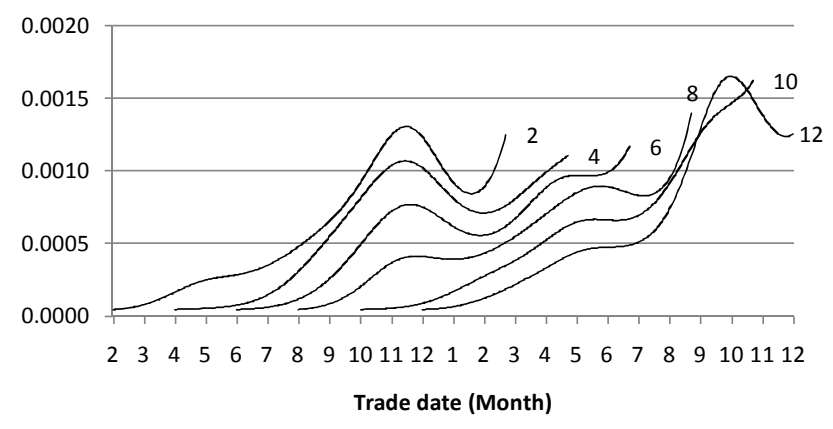

(c) Standard deviation of idiosyncratic error

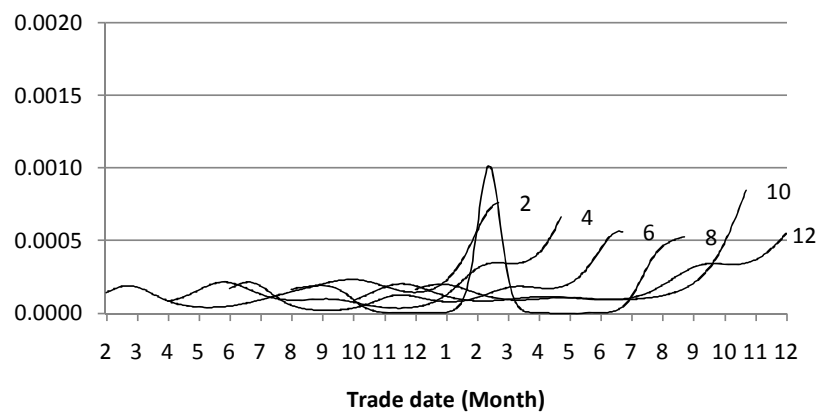

(d) Share of total variance accounted for by two common factors

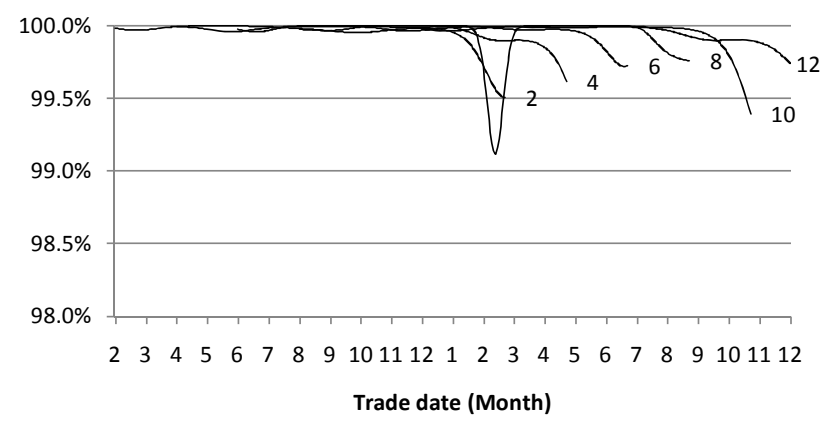


Figure 5. Factor loadings, variance of idiosyncratic error, and share of total variance accounted for by two common factors in conventional two-factor model: Natural gas

(a) Variance attributable to two common factors

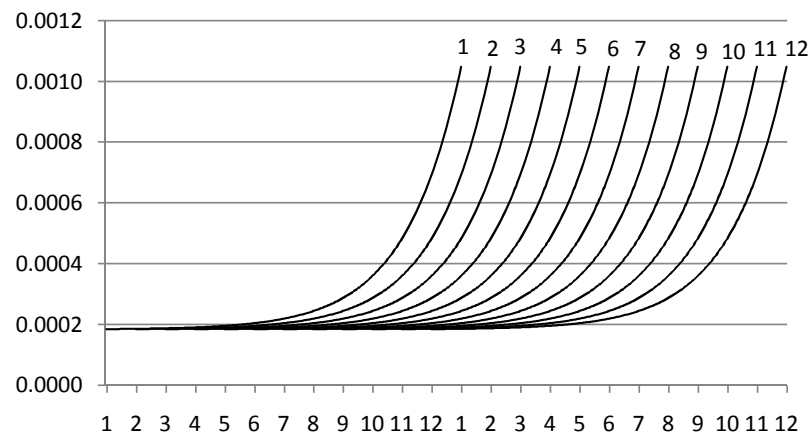

(b) Variance attributable to idiosyncratic error

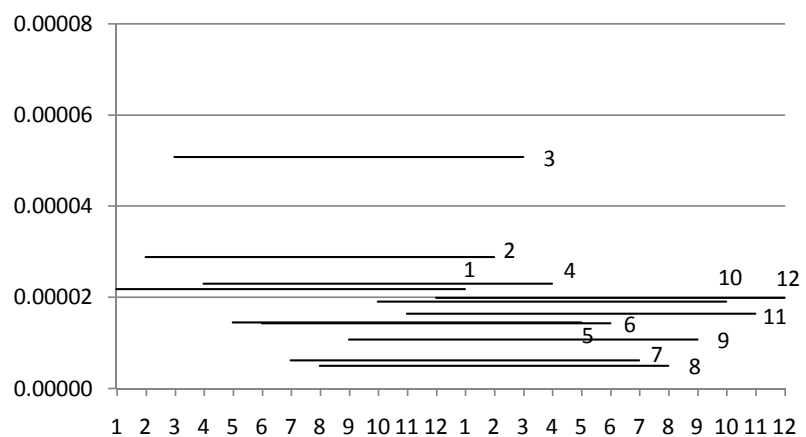

(c) Share of varaince accounted for by two common factors

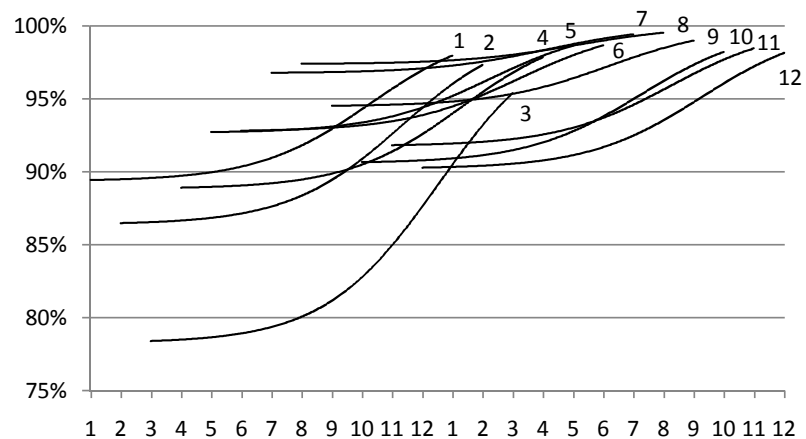


Figure 6. Factor loadings, variance of idiosyncratic error, and share of total variance accounted for by two common factors in conventional two-factor model: Corn

(a) Variance attributable to two common factors

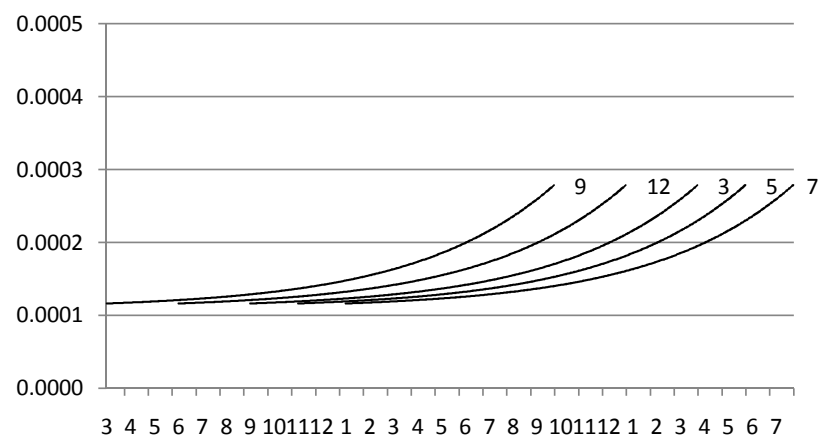

(b) Variance attributable to idiosyncratic error

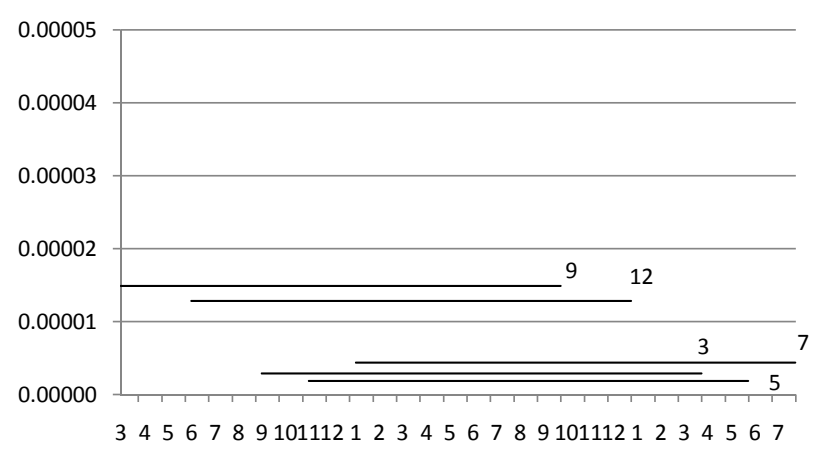

(c) Share of varaince accounted for by two common factors

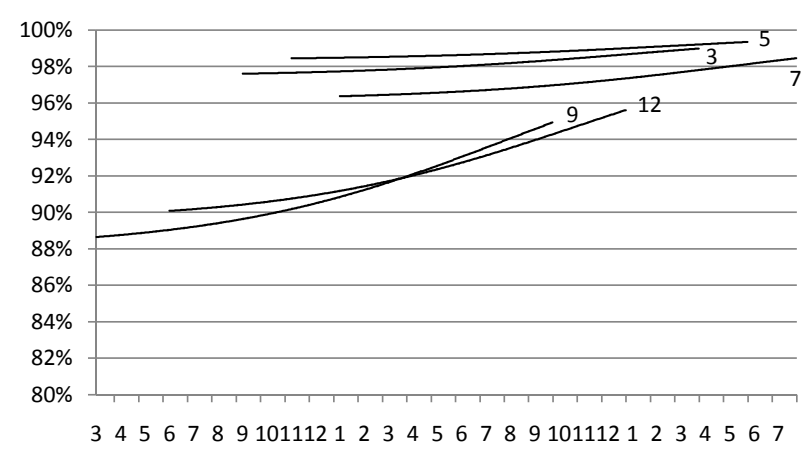


Figure 7. Factor loadings, variance of idiosyncratic error, and share of total variance accounted for by two common factors in conventional two-factor model: Crude oil

(a) Variance attributable to two common factors

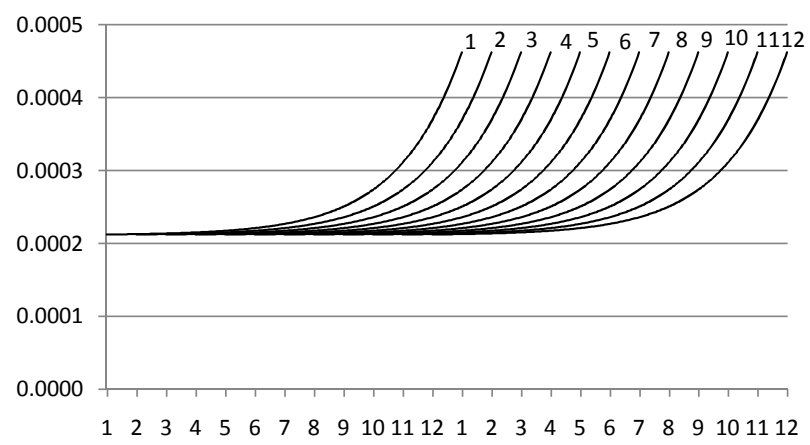

(b) Variance attributable to idiosyncratic error

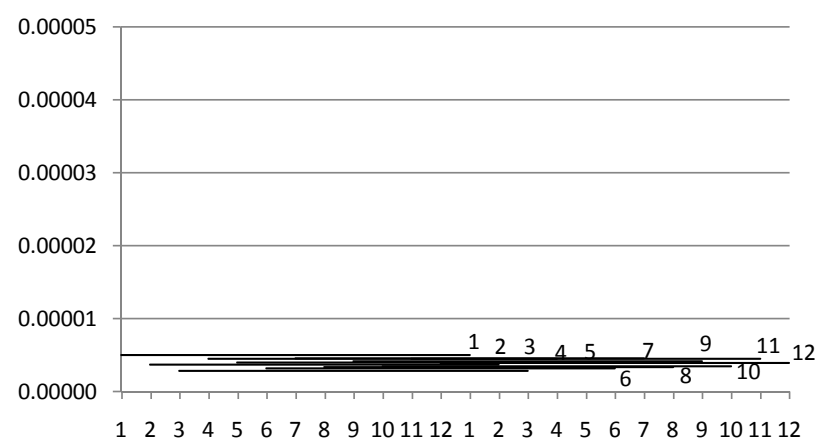

(c) Share of varaince accounted for by two common factors

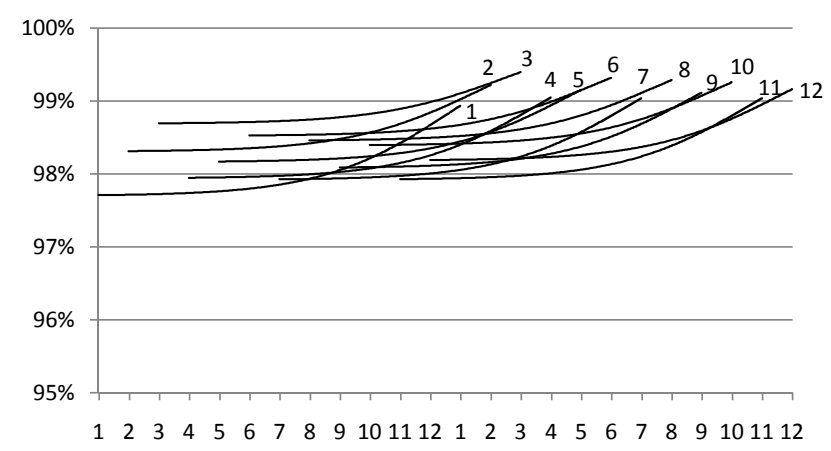


Figure 8. Factor loadings, variance of idiosyncratic error, and share of total variance accounted for by two common factors in conventional two-factor model: Gold

(a) Variance attributable to two common factors

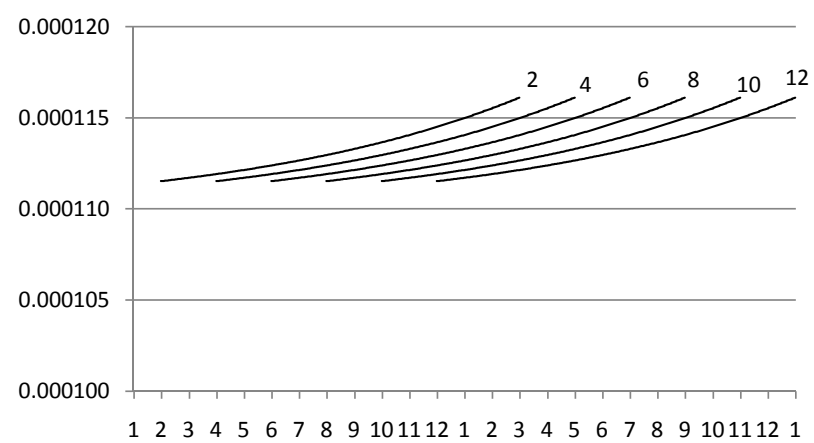

(b) Variance attributable to idiosyncratic error

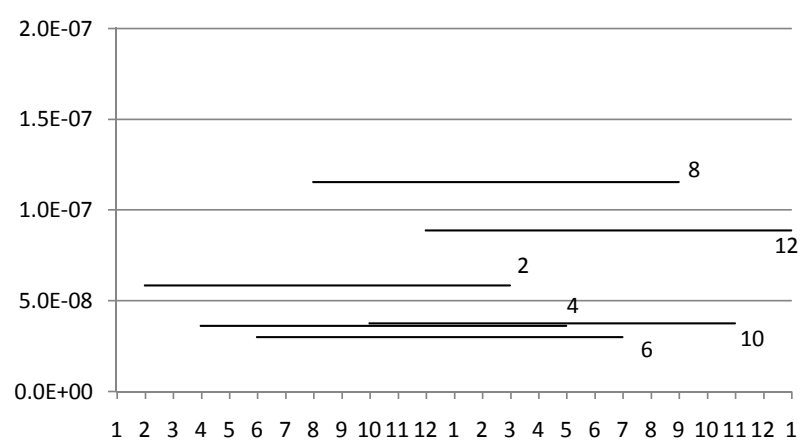

(c) Share of varaince accounted for by two common factors

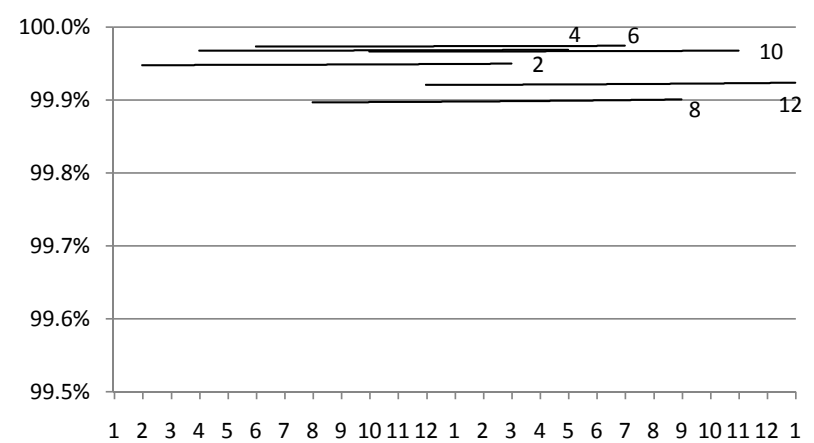


Figure 9. Factor loadings, variance of idiosyncratic error, and share of total variance accounted for by two common factors in the composite model: Natural gas

(a) Variance attributable to two common factors

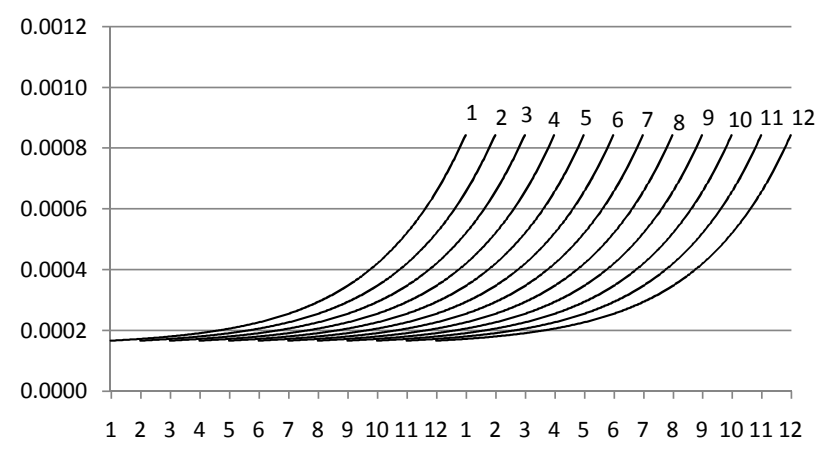

(b) Variance attributable to idiosyncratic error

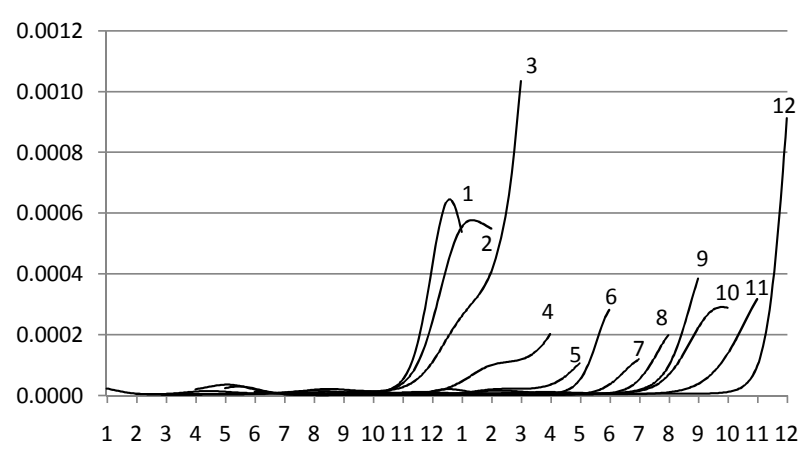

(c) Share of varaince accounted for by two common factors

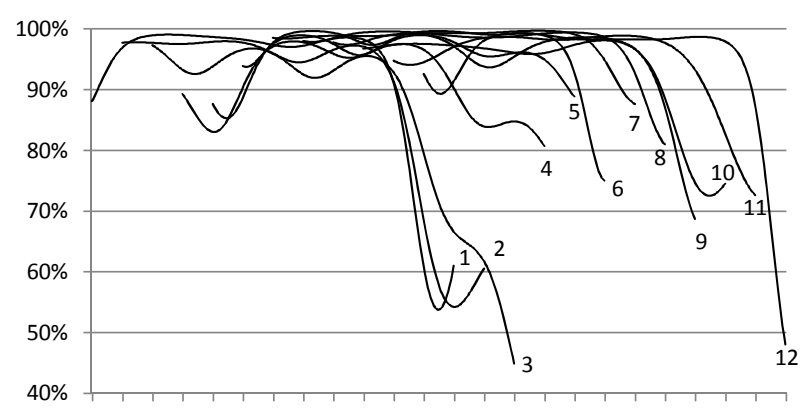

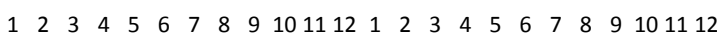


Figure 10. Factor loadings, variance of idiosyncratic error, and share of total variance accounted for by two common factors in the composite model: Corn

(a) Variance attributable to two common factors

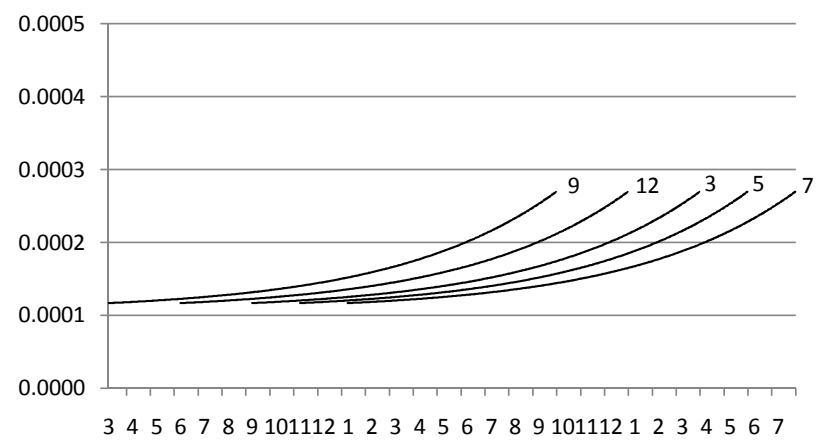

(b) Variance attributable to idiosyncratic error

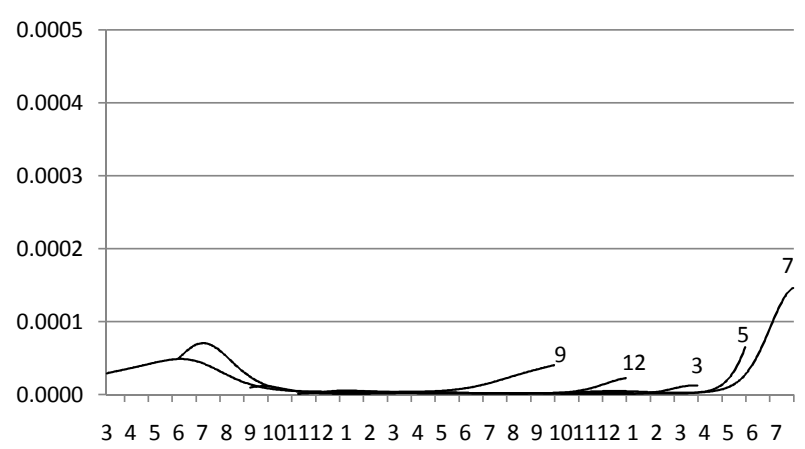

(c) Share of varaince accounted for by two common factors

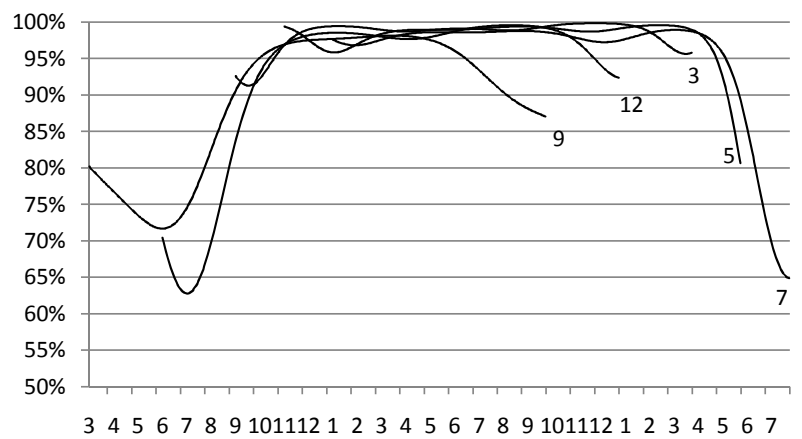


Figure 11. Factor loadings, variance of idiosyncratic error, and share of total variance accounted for by two common factors in the composite model: Crude oil

(a) Variance attributable to two common factors

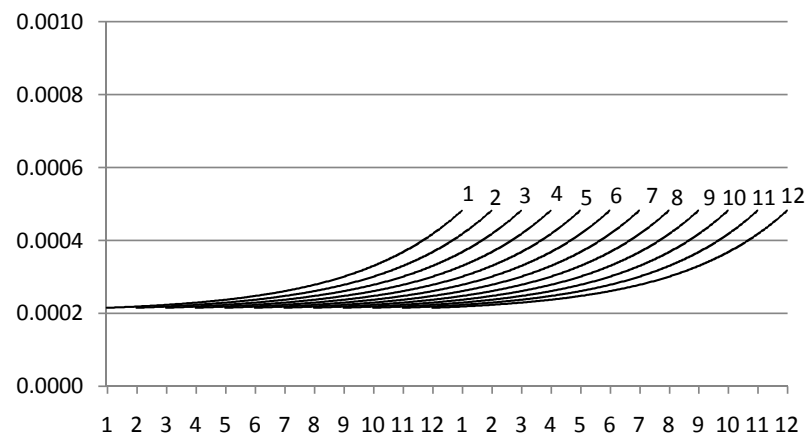

(b) Variance attributable to idiosyncratic error

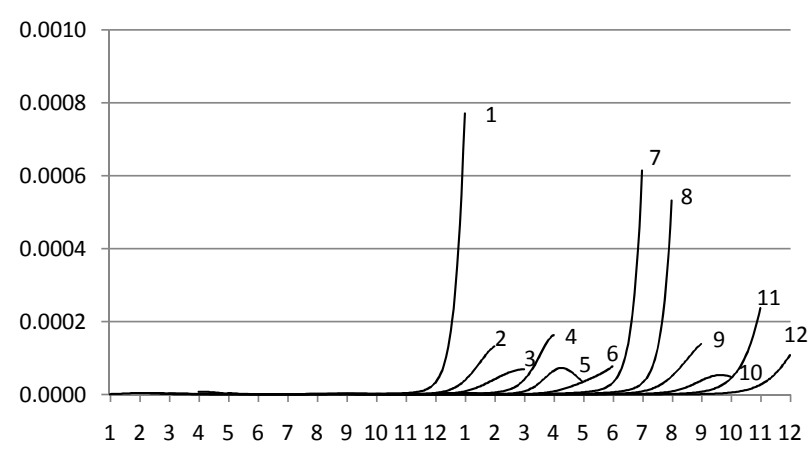

(c) Share of varaince accounted for by two common factors

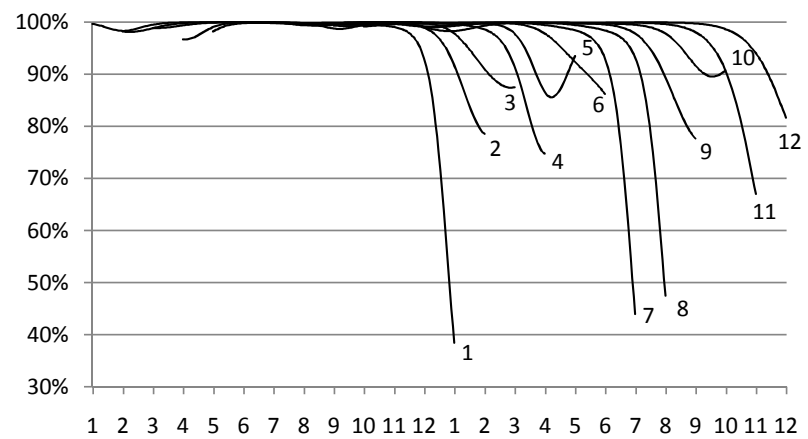


Figure 12. Factor loadings, variance of idiosyncratic error, and share of total variance accounted for by two common factors in the composite model: Gold

(a) Variance attributable to two common factors

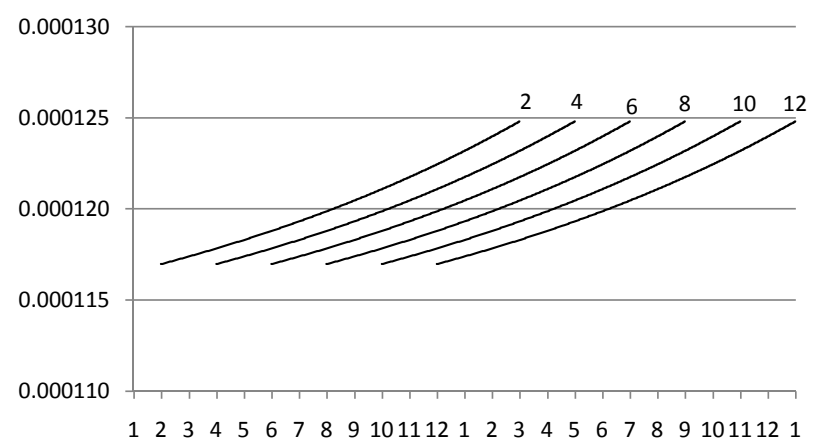

(b) Variance attributable to idiosyncratic error

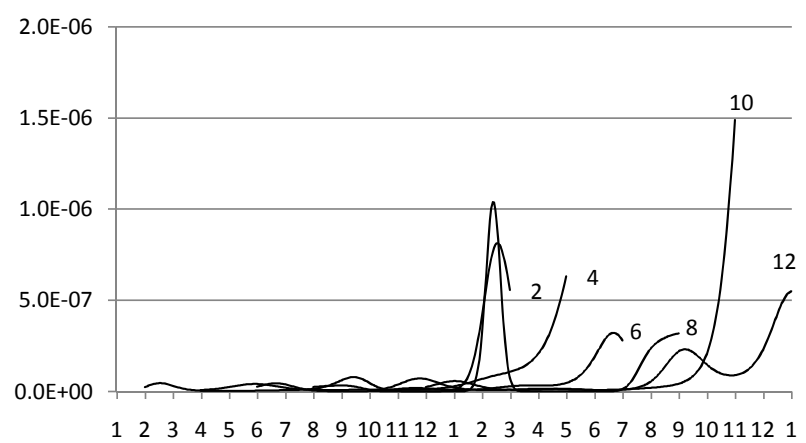

(c) Share of varaince accounted for by two common factors

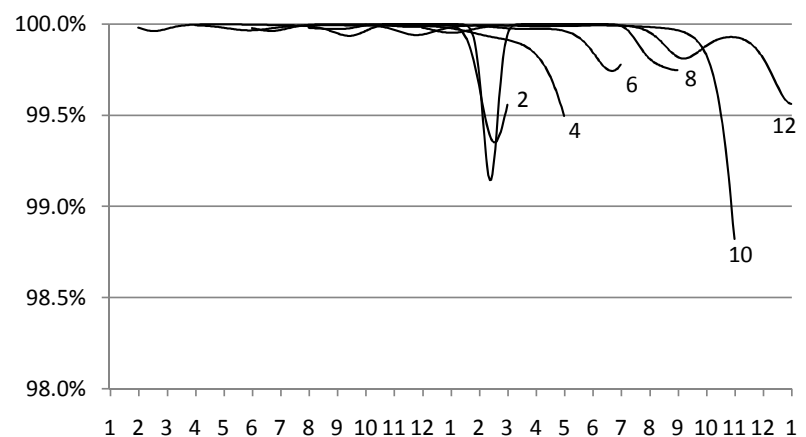


Figure 13. Position of contract included into the variance minimizing portfolio realtive to the spot (nearby futures) position

(a) Crude oil

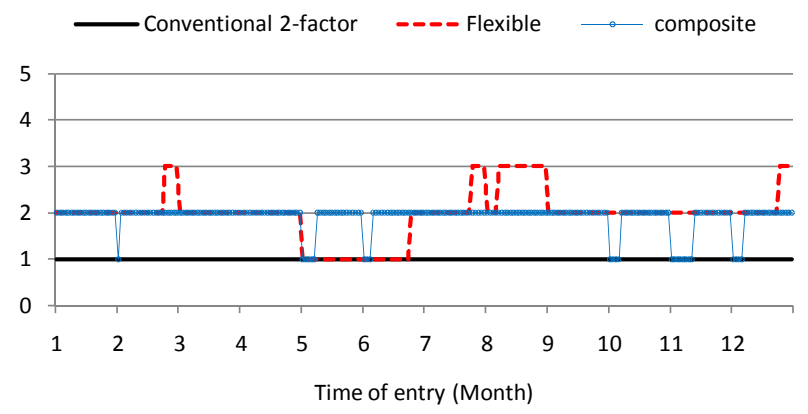

(b) Natural gas

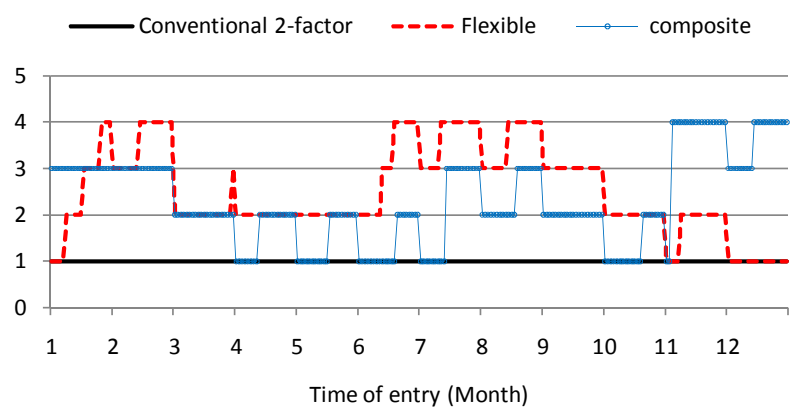

(c) Gold

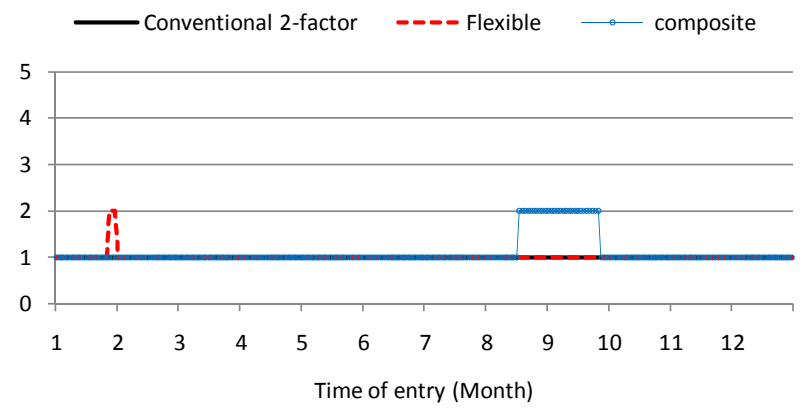

(d) Corn

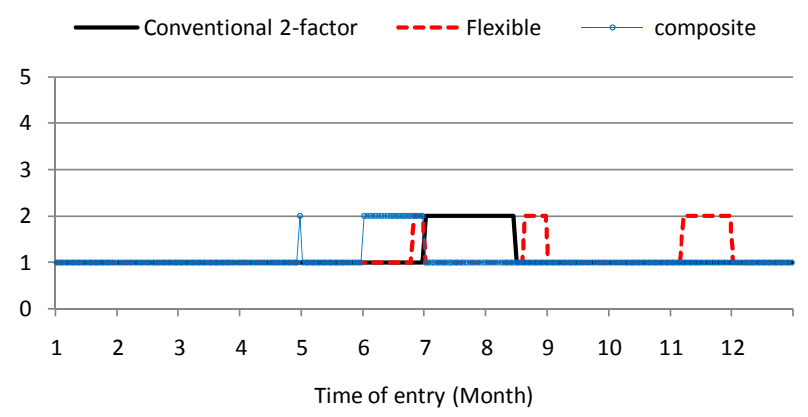


Figure 14. Optimal hedge ratio

(a) Crude oil

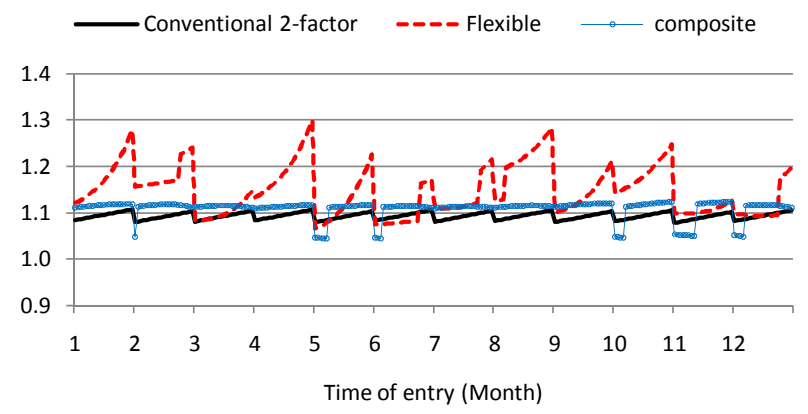

(b) Natural gas

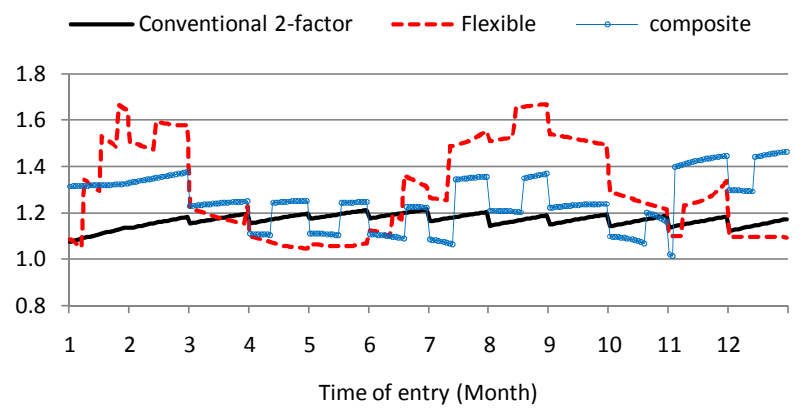

(c) Gold

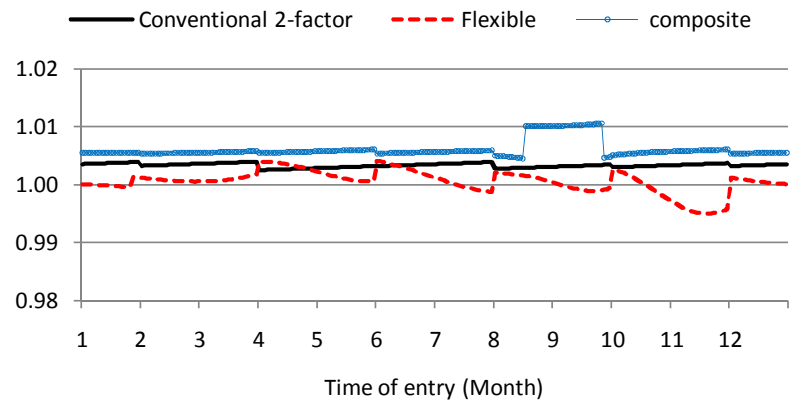

(d) Corn

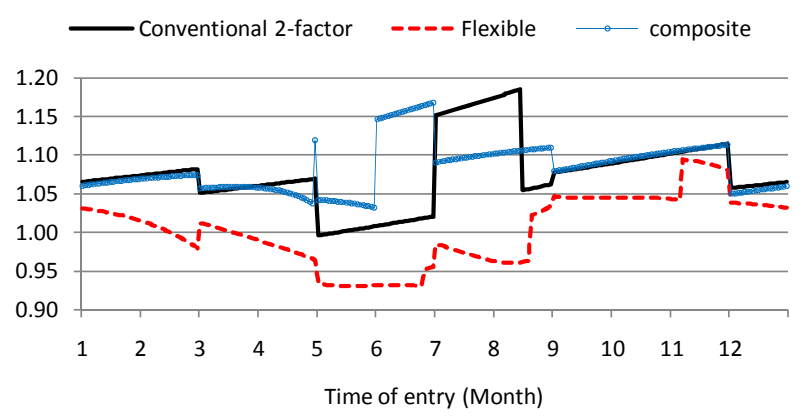

